\title{
Pure point spectrum for the time-evolution of a periodically rank-N kicked Hamiltonian
}

\author{
James McCaw* and B. H. J. McKellar ${ }^{\dagger}$ \\ School of Physics, Research Centre for High Energy Physics, \\ The University of Melbourne, Victoria, 3010, Australia.
}

(Dated: July 10, 2018)

\begin{abstract}
We find the conditions under which the spectrum of the unitary timeevolution operator for a periodically rank-N kicked system remains pure point. This stability result allows one to analyse the onset of, or lack of chaos in this class of quantum mechanical systems, extending the results for rank-1 systems produced by Combescure and others. This work includes a number of unitary theorems equivalent to those well known and used in the self-adjoint theory.
\end{abstract}

\section{Introduction}

We will derive conditions on the time-periodic perturbations to the base Hamiltonian for the spectrum of the Floquet operator to remain pure point. We consider Hamiltonians of the form

$$
H(t)=H_{0}+A^{*} W A \sum_{n=0}^{\infty} \delta(t-n T)
$$

where $A$ is bounded, $W$ is self adjoint and $H_{0}$ has pure point (discrete) spectrum. The time-evolution of such Hamiltonians is of great interest in quantum chaos, and of central importance is the spectral properties of the Floquet operator, defined as

$$
V=e^{i A^{*} W A / \hbar} e^{-i H_{0} T / \hbar}
$$

\footnotetext{
*Electronic mail: j.mccaw@physics.unimelb.edu.au
}

†Electronic mail: b.mckellar@physics.unimelb.edu.au 
which comes directly from considering the time-evolution of the kicked system

$$
U(t)=\left[\exp \left(-\frac{i}{\hbar} \int_{0}^{t} d t^{\prime} H\left(t^{\prime}\right)\right)\right]_{+}
$$

with $H\left(t^{\prime}\right)$ given by (1). The spectrum of the Floquet operator is known as the "quasi-energy spectrum".

This work is an extension of a result of Combescure [1]. Our results are based on the self-adjoint work by Howland [2]. If we choose $A$ to be a rank-1 perturbation,

$$
\begin{aligned}
A & =|\psi\rangle\langle\psi| \\
W & =\lambda I
\end{aligned}
$$

we reproduce the work of Combescure [1]. The vector $|\psi\rangle$ is a linear combination of orthonormal basis states, $\left|\phi_{n}\right\rangle$ of the unperturbed Hamiltonian $H_{0}$

$$
|\psi\rangle=\sum_{n=0}^{\infty} a_{n}\left|\phi_{n}\right\rangle
$$

Combescure showed that if $\psi \in l_{1}\left(H_{0}\right)$, that is if

$$
\sum_{n=0}^{\infty}\left|a_{n}\right|<\infty
$$

then the quasi-energy spectrum remains pure point for almost every perturbation strength $\lambda$. We will generalise this result to all finite rank perturbations

$$
\begin{aligned}
A & =\sum_{k=1}^{N} A_{k}=\sum_{k=1}^{N}\left|\psi_{k}\right\rangle\left\langle\psi_{k}\right| \\
W & =\sum_{k=1}^{N} \lambda_{k}\left|\psi_{k}\right\rangle\left\langle\psi_{k}\right|
\end{aligned}
$$

where $\lambda_{k} \in \mathbb{R}$ and each vector $\left|\psi_{k}\right\rangle$ is a linear combination of the $H_{0}$ basis states, $\left|\phi_{n}\right\rangle$

$$
\left|\psi_{k}\right\rangle=\sum_{n=0}^{\infty}\left(a_{k}\right)_{n}\left|\phi_{n}\right\rangle .
$$

The states $\left|\psi_{k}\right\rangle$ are orthogonal

$$
\left\langle\psi_{k} \mid \psi_{l}\right\rangle=\delta_{k l} .
$$


The basic result is that if each $\left|\psi_{k}\right\rangle$ is in $l_{1}\left(H_{0}\right)$, the spectrum of $V$ will remain pure point for almost every perturbation strength.

The perturbation for which we prove that the quasi-energy spectrum remains pure point is in fact more general than the finite rank perturbation presented above. The finite rank result is however the motivation for undertaking this work.

Howland [2] showed that the Hamiltonian (1) has a pure point spectrum if the $\psi_{k} \mathrm{~s}$ are in $l_{1}\left(H_{0}\right)$. Here, we follow a similar argument, showing that the continuous part of the spectrum of $V$ is empty, allowing us to conclude that the spectrum of $V$ must be pure point.

Associated with what we have termed the Floquet Operator, is the "Floquet Hamiltonian"

$$
K=-i d / d t+H(t)
$$

It turns out that $K$ provides a different way to access similar information to what we are seeking. Developed in papers by Howland [3], [4] and [5] and linked to our Floquet operator in [6] (p. 808), $K$ was introduced, in some part, because directly working with $V$ proved too difficult. The large body of knowledge on self-adjoint operators provides a mature basis for proving theorems about $K$. As discussed in [6], the spectrum of $K$ is easily related algebraically to that of $V$, so results on the spectrum for $K$ and $V$ are equivalent.

Working directly with $V$, however, as we do here, is valuable in that it gives a transparent, direct insight into the dynamics of the perturbed system $H(t)$. After the completion of this work, which is a unitary equivalent to that of Howland [2], we discovered that Howland had used his work [2] on the spectrum of self-adjoint operators to obtain similar results to what we do here [5].

The relationship between our work and Howland's works [2] and [5] is similar to the relationship between the self-adjoint rank-1 work of Simon and Wolff [7] and the unitary rank-1 work of Combescure [1].

The techniques developed in this paper provide new, general theorems applicable to unitary operators and show that it is possible to develop the theory of the spectrum of time-evolution operators directly, without need for the techniques of [3] briefly mentioned here.

\section{I.1 Motivation}

The classical study of chaos is now a well established and flourishing field of research in mathematics and mathematical physics. Chaotic behaviour seems to pervade a vast spectrum of dynamical systems, and an appreciation of it is essential for a detailed understanding of such systems. The classic example of the earth's weather patterns always comes to mind when chaos is mentioned. 
The microscopic world, however, is not governed by the laws of classical dynamics. In the realm of small quantum numbers the dynamics of a system is governed by the Schrödinger equation. In such systems, the simple and elegant definitions of chaos such as positive Lyapunov exponent, which hold for classical systems, are not applicable. In fact, there is no universally accepted definition of quantum chaos. Some model systems show what many would consider "chaotic behaviour", yet there are general arguments made by some [8] to the effect that "quantum chaos" does not exist. Our study of one aspect of "quantum chaos" is motivated by much of this work. Taking note of these uncertainties and conflicting views, two questions arise that are of central importance.

(a) What properties of a quantum mechanical system determine whether or not the corresponding classical system that derives from it will display chaotic behaviour?

(b) Are there in fact quantum systems that display chaotic behaviour at the quantum level?

The former question is intimately linked to the "Correspondence Principle" and theories of quantum measurement. Needless to say, this area of fundamental physics is infamous for its interpretational difficulties and seemingly inconsistent behaviour.

The latter question too, is the source of much debate in the literature. As in any immature study, quantum chaos is struggling to be self consistently defined. A wide range of possible definitions and interpretations of what quantum chaos actually is have been put forward, many in direct contradiction with one another. At some stage in the future presumably, we will find a satisfactory criteria for what constitutes quantum chaos. Until then, many attempts to look at particular aspects of the dynamics of quantum systems will be (and have been) made. Some papers, courtesy of their definition of quantum chaos, come to the conclusion that there is no such thing as quantum chaos. That is, they conclude that no quantum system can display chaotic behaviour. Other papers, simply as a consequence of a different starting point, come to the conclusion that there are quantum systems that display chaos.

There are general arguments that allow one to categorise the behaviour of a quantum system based upon the spectral composition of the quasi-energy spectrum. Hence, Combescure's work on the spectrum is relevant to the study of chaos. Our work, by extending the result of Combescure, may allow for the further categorisation of classes of Hamiltonian systems as chaotic or otherwise. This is further discussed in the next section. 


\section{I.2 Spectral analysis of operators and a link to chaos}

The intuitive definition for the energy spectrum of a quantum system is best seen through example, say the hydrogen atom. The bound states of hydrogen are a countable number of isolated, discrete energies. Each energy corresponds to an eigenvalue of the system and the set of these points makes up the point energy spectrum. The positive energy scattering states form the continuous energy spectrum. Thus, the energy spectrum for the hydrogen system consists of two disjoint parts: the negative energy discrete (or "point") spectrum, and the positive energy continuous spectrum.

For hydrogen, $\sigma_{p}(H)=\left\{\alpha_{n} ; \alpha_{n} \approx-13.6 / n^{2}\right.$ for $\left.n \in \mathbb{N}\right\}$, and $\sigma_{\text {cont }}(H)=$ $(0, \infty)$.

As another simple example, the harmonic oscillator quantum system has only discrete energy levels, and thus is said to be "pure point". That is, the eigenvectors of the harmonic oscillator form a basis of the Hilbert Space.

While these simple examples have shown clearly that we can split the energy spectrum into point and continuous parts, this is not the whole story. The mathematical treatment of operators and measures shows that the spectrum in fact consists of three parts, the point, absolutely continuous and singularly continuous spectrum. For an appreciation of the work that follows, a mathematically rigorous understanding of the spectra is necessary. The introductory chapters in [9] are essential reading.

One must note that the concept of spectrum is associated with a particular operator. Typically, we talk of the energy spectrum, associated with the Hamiltonian. However, all operators (e.g., Hamiltonian, Floquet etc.) have a spectrum. A failure to realise this has lead to a number of confused papers (see for example [10]) which use results on the spectrum of the Hamiltonian in a discussion of the spectrum of the Floquet operator. With these words of warning, we return to a discussion of the Floquet (or quasi-energy) spectrum of a quantum system.

The link between spectral properties and dynamics is an active field of research and is not yet fully understood. The introduction to the paper of Y. Last [11] provides an informative overview of the field and gives details on some of the most relevant theorems and results, including the RAGE theorem ([12], p. 341, Theorem XI.115). See also [13]. Y. Last's paper deals with systems where the Hamiltonian spectrum is of interest. In time-periodic Hamiltonian systems, the spectrum of the Floquet operator takes over that role. K. Yajima and H. Kitada [14] show that RAGE-like results apply to time-periodic systems, as we have here, and thus an analysis of the Floquet operator spectrum is of interest.

Refering to either the Hamiltonian spectrum or the Floquet spectrum where appropriate, and the appropriate RAGE-like theorem, we now comment on the "typical" manifestation of the spectrum. A typical quantum mechanical system 
does not posses a singularly continuous spectral component and thus, singular continuity is not usually mentioned in texts on quantum mechanics. This however, is not to say that it can't exist, or that it doesn't manifest itself in the dynamical behaviour of appropriate systems. With an understanding that Milek and Seba meant to refer to the RAGE-like theorem in [14] rather than the RAGE theorem itself, the argument presented in Section II of their paper [10] shows that if a system possesses a singularly continuous quasi-energy spectrum then its energy growth over time may be characteristic of a classically chaotic system. Thus, establishing the existence or otherwise of singular continuous spectra for the Floquet operator can be seen as of central importance to the question of whether or not a quantum mechanical system is chaotic. It must be noted that the arguments presented by Milek and Seba are acknowledged to be anything but rigorous - a point clearly established by Antoniou and Suchanecki [15, 16].

It is with the application of the RAGE-like theorem in mind [14], that we undertook the following work on the analysis of the quasi-energy spectrum of the class of Hamiltonians as defined by (1). The aforementioned work by Milek and Seba [10], utilising the rank-1 work of Combescure, has shown the manifestation of singularly continuous spectra in numerical simulations of rank-1 kicked rotor quantum systems. The work here has the potential to extend upon this, and provide a rigorous mathematical basis to numerical calculations on the time-evolution of higher rank kicked quantum systems.

\section{I.3 Outline and summary of results}

In Section [1] we will present the main theorems of the paper, concerned with establishing when systems of the form given by (10) maintain a pure point quasienergy spectrum. Parallelling Howland's paper [2] on self-adjoint perturbations of pure point Hamiltonians, the key ideas are those of $U$-finiteness and the absolute continuity of the multiplication operator $\mathbb{V}$. To establish the second of these concepts for our unitary case (remember that we are concerned with the spectral properties of the unitary time-evolution operator and not with the spectral properties of the self adjoint Hamiltonian itself), we require a modified version of the Putnam-Kato theorem [17]. This, and associated theorems are the topic of Section $\amalg$ Section \V uses the results of Section $\amalg$ and Section $\amalg$ to give the final results, which are then discussed in Section $\nabla$

\section{I.4 Notation}

We inherit our notation directly from the work of Howland [2]. $\mathcal{H}$ and $\mathcal{K}$ will denote Hilbert spaces throughout this paper. They will always be separable. The 
inner product of two vectors $x$ and $y$ is $\langle x, y\rangle$, and the norm of a vector $x$ is $\|x\|=\langle x, x\rangle^{1 / 2}$. For an operator $A: \mathcal{H} \rightarrow \mathcal{K}$ we define

- the domain $D(A)$; the vectors $x \in \mathcal{H}$ for which $A x$ is defined,

- the range $R(A)=\{y \in \mathcal{K}: y=A x$ for some $x \in \mathcal{H}\}$,

- the kernel $\operatorname{ker} A=\{x \in \mathcal{H}: A x=0\}$, and

- the operator norm $\|A\|=\sup _{x \in D(A):\|x\|=1}\{\|A x\|\}$.

For any set $S \in \mathbb{C}, \bar{S}$ is the closure of $S$. If $A_{n}$ is a sequence of operators, s$\lim A_{n}$ (also $A_{n} \stackrel{s}{\rightarrow} A$ ) denotes the strong limit, $\left\|\left(A_{n}-A\right) g\right\| \rightarrow 0$ for all $g \in \mathcal{H}$. w-lim $A_{n}$ (also $A_{n} \stackrel{w}{\rightarrow} A$ ) denotes the weak limit, $\left|\left\langle A_{n} g, f\right\rangle-\langle A g, f\rangle\right| \rightarrow 0$ for all $g, f \in \mathcal{H}$. By the Schwartz inequality, the weak limit exists if the condition above is satisfied for $f=g$. We will also have need for the norm limit of an operator, $\left\|A_{n}-A\right\| \rightarrow 0$.

For a unitary operator $V=\int e^{-i \theta} E(d \theta)$ on $\mathcal{H}$, we define for any Borel set $S$, $E[S]=\int_{S} E(d \theta)$. The $E(d \theta)$ are orthogonal projection operators, i.e., $E^{2}=E$ and thus

$$
\int|f(\theta)|^{2} E(d \theta)=\left|\int f(\theta) E(d \theta)\right|^{2} .
$$

We decompose our operator into its pure point $\left(V^{p}\right)$, singular continuous $\left(V^{s c}\right)$ and absolutely continuous $\left(V^{a c}\right)$ components. $V^{s}=V^{p}+V^{s c}$ is the singular part of the operator $V$. Similarly, we define the corresponding spectral measures $E^{p}$, $E^{s c}, E^{a c}$ and $E^{s}$. For a vector $x \in \mathcal{H}, m_{x}$ is the measure

$$
m_{x}(S)=\langle E(S) x, x\rangle .
$$

Again, we define $m_{x}^{p}, m_{x}^{s c}, m_{x}^{a c}$ and $m_{x}^{s}$. See ([9], p. 19-23) for an excellent description of these. By their definition, $m_{x}^{p}, m_{x}^{s c}$ and $m_{x}^{a c}$ are mutually singular, so we may write the Hilbert space as a direct sum (i.e., each of the spaces below is invariant)

$$
\mathcal{H}=\mathcal{H}_{p p} \oplus \mathcal{H}_{a c} \oplus \mathcal{H}_{s c} .
$$

The spectrum of $V$ is $\sigma(V)$, defined by

$$
\sigma(V)=\{\alpha \in \mathbb{C}: \alpha I-V \text { is not invertible }\} .
$$

If $T x=\alpha x$ for some $x \in \mathcal{H}$ and $\alpha \in \mathbb{C}$, then $x$ is an eigenvector, with corresponding eigenvalue $\alpha$. The closure of the set of $\alpha$ s forms the point spectrum of $\checkmark$

$$
\begin{aligned}
\sigma_{p}(V) & =\overline{\{\alpha: \alpha \text { an eigenvalue of } V\}} \\
& =\sigma\left(V \uparrow \mathcal{H}_{p}\right) .
\end{aligned}
$$


We say that $V$ is pure point if and only if the eigenvectors of $V$ form a basis of $\mathcal{H}$. The absolutely (singularly) continuous spectrum, $\sigma_{a c(s c)}(V)$ is similarly defined by

$$
\sigma_{a c(s c)}(V)=\sigma\left(V\left\lceil\mathcal{H}_{a c(s c)}\right) .\right.
$$

For a complete discussion and analysis of these topics, the most convenient reference is ([9], p. 19-23, 188, 230-231) or [18].

A set $\mathcal{S} \in \mathcal{H}$ is said to reduce an operator $A$ if both $\mathcal{S}$ and its ortho-complement $\mathcal{H} \ominus \mathcal{S}$ are invariant subspaces for $A$.

A vector $\phi$ is cyclic for an operator $A$ if and only if finite linear combinations of elements of $\left\{A^{n} \phi\right\}_{n=0}^{\infty}$ are dense in $\mathcal{H}$. This motivates the definition that a set $\mathcal{S}$ is cyclic for $\mathcal{H}$ if and only if the smallest closed reducing subspace of $\mathcal{H}$ containing $\mathcal{S}$ is $\mathcal{H}$.

We will also use some basic set notation. $A \cap B$ and $A \cup B$ are, as usual, the intersection and union of sets $A$ and $B$ respectively. $A^{\mathrm{c}}$ is the complement of $A$. $A \sim B$ is $A \cap B^{\mathrm{c}}$. Note that $(A \sim B) \sim C$ is not equal to $A \sim(B \sim C)$, the former being a subset of the latter.

The function $\chi_{S}(x)$ is the characteristic function for a set $S$.

\section{Spectral properties of the Floquet operator}

Let $U$ be unitary on $\mathcal{H}$ and let $\mathcal{K}$ be an auxiliary Hilbert space. Define the closed operator $A: \mathcal{H} \rightarrow \mathcal{K}$, with dense domain $D(A)$. For our purposes, $A$ bounded on $\mathcal{H}$ is adequate. We work with a modification (multiplication by $e^{i \theta}$ ) of the resolvent of $U$

$$
F(\theta ; U)=\left(1-U e^{i \theta}\right)^{-1}
$$

and define for $\theta \in[0,2 \pi)$, and $\epsilon>0$ the function $G_{\epsilon}: \mathcal{K} \rightarrow \mathcal{K}$

$$
G_{\epsilon}(\theta ; U, A)=A F^{*}\left(\theta_{+} ; U\right) F\left(\theta_{+} ; U\right) A^{*} .
$$

where $\theta_{ \pm}=\theta \pm i \epsilon$. Let $J$ be a subset of $[0,2 \pi)$.

DEFINITION II.1 (U-FINITE) The operator $A$ is $U$-finite if and only if the operator $G_{\epsilon}(\theta ; U, A)$ has a bounded extension to $\mathcal{K}$, and

$$
G(\theta ; U, A)=\mathrm{s}-\lim _{\epsilon \downarrow 0} G_{\epsilon}(\theta ; U, A)
$$

exists for a.e. $\theta \in J$. 
We define the function

$$
\begin{aligned}
\delta_{\epsilon}(t) & =\frac{1}{2 \pi}\left(\sum_{n=0}^{\infty} e^{i n(t+i \epsilon)}+\sum_{n=-\infty}^{0} e^{i n(t-i \epsilon)}-1\right) \\
& =\frac{1}{2 \pi} \frac{1-e^{-2 \epsilon}}{1-2 e^{-\epsilon} \cos (t)+e^{-2 \epsilon}} .
\end{aligned}
$$

The limit as $\epsilon \rightarrow 0$ of $\delta_{\epsilon}(t)$ is a series representation of the $\delta$-function. The proof is based on showing that

$$
\lim _{\epsilon \downarrow 0} \int_{-\pi}^{\pi} g(t) \delta_{\epsilon}(t) d t=0
$$

where $g(t)=f(t)-f(0)$ and $f(t)$ is bounded in $(-\pi, \pi)$. One splits the integral into three parts, $\int_{-\pi}^{-\xi}+\int_{-\xi}^{\xi}+\int_{\xi}^{\pi}$. One must assume that $f(t)$ is continuous at $t=0$ (otherwise $\int f(t) \delta(t) d t$ is not well defined) so that

$$
\forall \eta, \exists \xi>0 \text { s.t. } \forall t,|t|<\xi \text { we have }|f(t)-f(0)|<\eta \text {. }
$$

The assumption that $f(t)$ is bounded on $(-\pi, \pi)$ is also required.

The first and third integrals are zero because $\delta_{\epsilon}(t) \rightarrow 0$ for $t \neq 0$ from (11) and the assumption that $g(t)$ is bounded. The second integral from $-\xi$ to $\xi$ is zero by the continuity of $f(t)$ at $t=0$, the positivity of $\delta_{\epsilon}(t)$ and ([19], p. 435, (3.792.1)).

Given (11) and the spectral decomposition of $U$, we may write

$$
\begin{aligned}
\delta_{\epsilon}\left(1-U e^{i \theta}\right) & =\int \delta_{\epsilon}\left(1-e^{i\left(\theta-\theta^{\prime}\right)}\right) E\left(d \theta^{\prime}\right) \\
& =\int \delta_{\epsilon}\left(\theta-\theta^{\prime}\right) E\left(d \theta^{\prime}\right) \\
& =\frac{1}{2 \pi}\left(1-e^{-2 \epsilon}\right) F^{*}\left(\theta_{+} ; U\right) F\left(\theta_{+} ; U\right) .
\end{aligned}
$$

The existence of a non-trivial $U$-finite operator will have important consequences for the spectrum of the Floquet operator $V$. We introduce the set

$$
N(U, A, J)=\left\{\theta \in J: \mathrm{s}-\lim _{\epsilon \downarrow 0} G_{\epsilon}(\theta ; U, A) \text { does not exist }\right\}
$$

of measure zero, which enters the theorem. We will often refer to this set simply as $N$ during proofs.

THEOREM II.2 If $A$ is $U$-finite on $J$ and $R\left(A^{*}\right)$ is cyclic for $U$, then

(a) U has no absolutely continuous spectrum in $J$, and 
(b) the singular spectrum of $U$ in $J$ is supported by $N(U, A, J)$.

Proof. (国) Following Howland, we note that the absolutely continuous spectral measure, $m_{y}^{a c}(J)$ is the $\epsilon \rightarrow 0$ limit of $\left\langle\delta_{\epsilon}\left(1-U e^{i \theta}\right) y, y\right\rangle$ for $\theta \in J$. If $y \in \mathcal{H}$ is in $R\left(A^{*}\right)$, allowing us to write $y=A^{*} x$ for some $x \in \mathcal{K}$, then

$$
\begin{aligned}
\lim _{\epsilon \downarrow 0} & \left\langle\delta_{\epsilon}\left(1-U e^{i \theta}\right) y, y\right\rangle \\
& =\lim _{\epsilon \downarrow 0}\left\langle\delta_{\epsilon}\left(1-U e^{i \theta}\right) A^{*} x, A^{*} x\right\rangle \\
& =\lim _{\epsilon \downarrow 0} \frac{\epsilon(1-\epsilon)}{\pi}\left\langle G_{\epsilon}(\theta ; U, A) x, x\right\rangle=0
\end{aligned}
$$

for a.e. $\theta \in J$. The set $\mathcal{Y}$ of vectors $y$ for which $m_{y}^{a c}(J)=0$ is a closed reducing subspace of $\mathcal{H}$, and by construction contains the cyclic set $R\left(A^{*}\right)$ as a subset. Because $\mathcal{Y}$ is invariant, finite linear combinations of action with $U^{n}$ leaves us in $\mathcal{Y}$. Due to the cyclicity, these same linear combinations allow us to reach any $y \in \mathcal{H}$. Thus, the set $\mathcal{Y}$ of vectors $y$ with $m_{y}^{a c}(J)=0$ must be the whole Hilbert space $\mathcal{H}$. So there is no absolutely continuous spectrum of $U$ in $J$.

(b) A theorem of de la Vallée Pousin ([20], p. 127, (9.6)) states that the singular part of the spectrum of a function is supported on the set where the derivative is infinite. In our case, this corresponds to finding where $m_{y}(d \theta) \rightarrow \infty$. We calculate

$$
\begin{aligned}
\lim _{\epsilon \downarrow 0}\left\langle\delta_{\epsilon}\left(1-U e^{i \theta}\right) y, y\right\rangle & =\int \delta\left(\theta-\theta^{\prime}\right)\left\langle E\left(d \theta^{\prime}\right) y, y\right\rangle \\
& =\int \delta\left(\theta-\theta^{\prime}\right) m_{y}\left(d \theta^{\prime}\right) \\
& =m_{y}(d \theta) .
\end{aligned}
$$

Thus, $m_{y}^{s}=m_{y}^{s c}+m_{y}^{p p}$ is supported on the set where

$$
\lim _{\epsilon \downarrow 0}\left\langle\delta_{\epsilon}\left(1-U e^{i \theta}\right) y, y\right\rangle=\infty .
$$

From the proof to part (ail), if $y=A^{*} x$ then the limit (13) is zero for $\theta \in$ $J, \theta \notin N$, so $m_{y}^{s}$ in $J$ must be supported by $N$. The set of vectors $y$ with $m_{y}^{s}\left(J \cap N^{c}\right) \equiv m_{y}^{s}(J \sim N)=0$ is closed, invariant and contains $R\left(A^{*}\right)$, so must be $\mathcal{H}$ by the argument above. Thus, the singular spectrum of $U$ is supported on the set $N$.

We now define a new operator, $Q(z): \mathcal{K} \rightarrow \mathcal{K}$

$$
Q(z)=A(1-U z)^{-1} A^{*} \text {. }
$$


Note that

$$
Q\left(e^{i \theta_{ \pm}}\right)=A F\left(\theta_{ \pm} ; U\right) A^{*} .
$$

$Q(z)$ is clearly well defined for $|z| \neq 1$. Proposition $\llbracket .3$ shows that the definition can be extended to $|z|=1$.

Proposition II.3 Let $A$ be bounded. If $\theta \in J$, but $\theta \notin N(U, A, J)$, then

(a) the operator $Q\left(e^{i \theta}\right)=A\left(1-U e^{i \theta}\right)^{-1} A^{*}$ is bounded on $\mathcal{K}$, and

(b) one has s-lim $\lim _{\epsilon \downarrow 0}\left(e^{ \pm i(\theta \pm i \epsilon)}\right)=Q\left(e^{ \pm i \theta}\right)$.

Proof. (a) Without loss of generality, take $\theta=0(z=1)$. By Theorem $e^{-i 0} \notin \sigma_{p}(U)$, so $\left(1-U e^{i 0}\right)^{-1}$ exists as a densely defined operator. As $A$ is a bounded operator, it suffices to show that $\left(1-U e^{i 0}\right)^{-1} A^{*}$ is bounded. We have

$$
\begin{aligned}
\left\|\left(1-U e^{i 0_{+}}\right)^{-1} A^{*} x\right\|^{2} & =\left\langle F\left(0_{+} ; U\right) A^{*} x, F\left(0_{+} ; U\right) A^{*} x\right\rangle \\
& =\left\langle A F^{*}\left(0_{+} ; U\right) F\left(0_{+} ; U\right) A^{*} x, x\right\rangle \\
& =\left\langle G_{\epsilon}(0 ; U, A) x, x\right\rangle \leq C|x|^{2}(\operatorname{as} \theta \notin N)
\end{aligned}
$$

for some real constant $C$. If $y=A^{*} x$, noting $U=\int e^{-i \theta} E(d \theta)$, we also have

$$
\left\|\left(1-U e^{i 0_{+}}\right)^{-1} A^{*} x\right\|^{2}=\int\left(\frac{1}{1-e^{-i \theta} e^{-\epsilon}}\right)\left(\frac{1}{1-e^{i \theta} e^{-\epsilon}}\right)\langle E(d \theta) y, y\rangle .
$$

In light of (15), we may safely take $\epsilon$ to zero, to obtain

$$
\int\left(\frac{1}{1-e^{-i \theta}}\right)\left(\frac{1}{1-e^{i \theta}}\right)\langle E(d \theta) y, y\rangle \leq C\|x\|^{2}<\infty .
$$

From (16), we have

$$
\begin{aligned}
& \int\left(\frac{1}{1-e^{-i \theta}}\right)\left(\frac{1}{1-e^{i \theta}}\right)\langle E(d \theta) y, y\rangle \\
&=\left\langle[1-U]^{-1} y,[1-U]^{-1} y\right\rangle \leq C\|x\|^{2}<\infty
\end{aligned}
$$

so $y \in D\left[(1-U)^{-1}\right]$. Thus, $Q(1)=A(1-U)^{-1} A^{*}$ is defined on all $\mathcal{K}$ and bounded.

(b) For $y \in D\left((1-U)^{-1}\right)$, we show that the difference between $Q\left(e^{ \pm i(0 \pm i \epsilon)}\right)$ and $Q\left(e^{ \pm i 0}\right)$ tends to zero as $\epsilon \rightarrow 0$. Again, due to the boundedness of $A$, we need only show that

$$
\left\|\left(\left(1-U e^{i 0_{+}}\right)^{-1}-(1-U)^{-1}\right) A^{*} x\right\|
$$


tends to zero. Consider

$$
\begin{aligned}
& \left|\left(1-U e^{-\epsilon}\right)^{-1} y-(1-U)^{-1} y\right|^{2} \\
& =\int\left|\frac{1}{1-e^{-i \theta} e^{-\epsilon}}-\frac{1}{1-e^{-i \theta}}\right|^{2}\langle E(d \theta) y, y\rangle \\
& =\int\left(\frac{\left(1-e^{-\epsilon}\right)^{2}}{1-2 e^{-\epsilon} \cos \theta+e^{-2 \epsilon}}\right) \frac{\langle E(d \theta) y, y\rangle}{\left(1-e^{-i \theta}\right)\left(1-e^{i \theta}\right)} .
\end{aligned}
$$

The first factor is bounded and tends to zero for $\theta \neq 0$. The second factor is the measure from (16). Clearly, away from the origin, the integral tends to zero. About the origin, we must take some care to show that there is no contribution to the integral.

Using (11), we have

$$
\frac{\left(1-e^{-\epsilon}\right)^{2}}{1-2 e^{-\epsilon} \cos \theta+e^{-2 \epsilon}}=\frac{\left(1-e^{-\epsilon}\right)^{2}}{1-e^{-2 \epsilon}} 2 \pi \delta_{\epsilon}(\theta) .
$$

On substitution into [18, we obtain

$$
\frac{\left(1-e^{-\epsilon}\right)^{2}}{1-e^{-2 \epsilon}} 2 \pi \int_{-\alpha}^{\alpha} \delta_{\epsilon}(\theta) \frac{m_{y}(d \theta)}{2(1-\cos \theta)}=\frac{\left(1-e^{-\epsilon}\right)^{2}}{1-e^{-2 \epsilon}} 2 \pi \int_{-\alpha}^{\alpha} \frac{d \Theta_{\epsilon}}{d \theta} \frac{g_{y}(\theta)}{2(1-\cos \theta)} d \theta
$$

The function $\Theta_{\epsilon}(\theta)=\int \delta_{\epsilon}\left(\theta^{\prime}\right) d \theta^{\prime}$ is the step function in the $\epsilon \rightarrow 0$ limit. For non-zero $\epsilon$ it is positive, monotonic, increasing and bounded by unity. As $\theta \notin N$ we have also written $m_{y}(d \theta)=g_{y}(\theta) d \theta$ for some well behaved positive function $g_{y}(\theta)$. By integration by parts (see [21], p. 32 for existence conditions, which are satisfied) we obtain

$$
\frac{\left(1-e^{-\epsilon}\right)^{2}}{1-e^{-2 \epsilon}} 2 \pi\left\{\left[\Theta_{\epsilon}(\theta) \frac{g_{y}(\theta)}{2(1-\cos \theta)}\right]_{-\alpha}^{\alpha}-\int_{-\alpha}^{\alpha} \Theta_{\epsilon}(\theta) \frac{d}{d \theta} \frac{g_{y}(\theta)}{2(1-\cos \theta)} d \theta\right\} .
$$

The first term within the curly braces is clearly some finite value. The second term is less than

$$
\int_{-\alpha}^{\alpha} \frac{d}{d \theta} \frac{g_{y}(\theta)}{2(1-\cos \theta)} d \theta=\left[\frac{g_{y}(\theta)}{2(1-\cos \theta)}\right]_{-\alpha}^{\alpha}
$$

from the properties of the $\Theta_{\epsilon}$ function mentioned above. As with the first term, it is clearly some finite value. Noting that

$$
\lim _{\epsilon \downarrow 0} \frac{\left(1-e^{-\epsilon}\right)^{2}}{1-e^{-2 \epsilon}}=0
$$

we see that part (b) follows. 
THEOREM II.4 Let $A$ be bounded and U-finite on J, with $R\left(A^{*}\right)$ cyclic for $U$. Let $W$ be bounded and self-adjoint on $\mathcal{K}$, and define the Floquet operator

$$
V=e^{i A^{*} W A / \hbar} U \text {. }
$$

Assume that for $|z| \neq 1, Q(z)$ is compact, and that $Q\left(e^{ \pm i(\theta \pm i \epsilon)}\right)$ converges to $Q\left(e^{ \pm i \theta}\right)$ in operator norm as $\epsilon \rightarrow 0$ for a.e. $\theta$ in $J$. Define the set

$$
M(U, A, J)=\left\{\theta \in J: Q\left(e^{ \pm i(\theta \pm i 0)}\right) \text { does not exist in norm }\right\} .
$$

\section{Then}

(a) V has no absolutely continuous spectrum in $J$, and

(b) the singular continuous part of the spectrum of $V$ in $J$ is supported by the set $N(U, A, J) \cup M(U, A, J)$.

Proof. (a) For convenience, we write the Floquet operator as

$$
V=\left(1+A^{*} Z A\right) U
$$

where $Z$ is defined appropriately by requiring ${ }^{1} \exp \left(i A^{*} W A / \hbar\right)=1+A^{*} Z A$. Noting (8) and (14) allows us to define

$$
\begin{aligned}
Q_{1}\left(e^{i \theta}\right) & =A F(\theta ; V) A^{*} \\
& =A\left(1-V e^{i \theta}\right)^{-1} A^{*} .
\end{aligned}
$$

Consider some vector $y^{\prime} \in \mathcal{H} . A y^{\prime}=x \in \mathcal{K}$ is defined for such $y^{\prime} . A^{*} x=$ $y^{\prime \prime}$ is some vector in $\mathcal{H}$. The cyclicity of $R\left(A^{*}\right)$ means that action with linear combinations of powers of $U$ on $y^{\prime \prime}$ allows us to obtain any $y \in \mathcal{H}$, our original $y^{\prime}$ being one of them. Thus, we have a construction of $A^{-1}$, namely, operation with $A^{*}$ followed by the linear combination of powers of $U$. As $y^{\prime}$ was arbitrary, $A^{-1}$ exists for all $y \in \mathcal{H}$. This allows us to introduce $I=A^{-1} A$ in what follows 2 .

We now proceed by use of the resolvent equation

$$
\begin{aligned}
Q_{1} & -Q \\
& =A\left\{\frac{1}{1-V e^{i \theta}}-\frac{1}{1-U e^{i \theta}}\right\} A^{*} \\
& =A\left\{\frac{1}{1-V e^{i \theta}}\left(A^{*} Z A U e^{i \theta}\right) \frac{1}{1-U e^{i \theta}}\right\} A^{*} \\
& =Q_{1}\left(e^{i \theta}\right) Z A U A^{-1} e^{i \theta} Q\left(e^{i \theta}\right) .
\end{aligned}
$$

\footnotetext{
${ }^{1}$ For the rank-N perturbation case where $W=\sum_{k=1}^{N} \lambda_{k}\left|\psi_{k}\right\rangle\left\langle\psi_{k}\right|$ and $A=\sum_{k=1}^{N}\left|\psi_{k}\right\rangle\left\langle\psi_{k}\right|$, we have $Z=\sum_{k=1}^{N}\left(\exp \left(i \lambda_{k} / \hbar\right)-1\right)\left|\psi_{k}\right\rangle\left\langle\psi_{k}\right|$.

${ }^{2}$ The particular choice of $A$ as a projection in (5) does not have an inverse, but we will see in Section $\square$ that we can define a subspace of $\mathcal{H}$ on which $R\left(A^{*}\right)$ is cyclic, and apply this theorem.
} 
Thus, briefly using $L=Z A U A^{-1} e^{i \theta}$ for clarity, we have

$$
\begin{aligned}
& L Q_{1}-L Q=L Q_{1} L Q \\
& \Rightarrow \quad\left(1+L Q_{1}\right)(1-L Q)=1 \\
& \Rightarrow 1+e^{i \theta} Z A U A^{-1} Q_{1}\left(e^{i \theta}\right)= \\
& {\left[1-e^{i \theta} Z A U A^{-1} Q\left(e^{i \theta}\right)\right]^{-1} . }
\end{aligned}
$$

Denote by $N$ and $M$ the sets $N(U, A, J)$ and $M(U, A, J)$. If $\theta \in(J \sim N) \sim M$, i.e., $\theta \in J \cap N^{c} \cap M^{c}$, and $1-e^{i \theta} Z A U A^{-1} Q\left(e^{i \theta}\right)$ is not invertible, then the compactness of $-L Q\left(e^{i \theta}\right)$ (which follows from the compactness of $Q\left(e^{i(\theta+i \epsilon)}\right)$, the norm convergence of $Q\left(e^{i(\theta+i \epsilon)}\right)$ and Theorem VI.12 in [9]) allows us to use the Fredholm Alternative ([9], p. 201, Theorem VI.14) to assert that

$$
\exists x \in \mathcal{K} \text {, s.t. }\left[1-e^{i \theta} Z A U A^{-1} Q\left(e^{i \theta}\right)\right] x=0 .
$$

That is, there is some vector $x \in \mathcal{K}$ which satisfies the equation

$$
x-e^{i \theta} Z A U A^{-1} A\left(1-U e^{i \theta}\right)^{-1} A^{*} x=0 .
$$

As $\theta \in J \sim N$, by Proposition $\llbracket .3 y=A^{*} x \in D\left[\left(1-U e^{i \theta}\right)^{-1}\right]$ so define $\phi$ as

$$
\phi=\left(1-U e^{i \theta}\right)^{-1} A^{*} x
$$

$\phi$ is a well defined vector on $\mathcal{H}$ and we have

$$
x=e^{i \theta} Z A U \phi .
$$

By (22), $x \neq 0$ implies $\phi \neq 0$, so we have

$$
\begin{aligned}
\left(1-U e^{i \theta}\right) \phi=A^{*} x & =e^{i \theta} A^{*} Z A U \phi \\
\text { or } \quad V \phi & =e^{-i \theta} \phi .
\end{aligned}
$$

We conclude that $e^{-i \theta} \in \sigma_{p}(V)$.

The multiplicity of the eigenvalue is given by the dimension of the kernel of $1-e^{i \theta} Z A U A^{-1} Q$, which is finite by the compactness of $Q$ and Theorem 4.25 of [22].

Therefore, if $\theta \in J \sim\left(N \cup M \cup \sigma_{p}(V)\right)$, which is a set of full Lebesgue measure $^{3}$, then the vector

$$
\begin{aligned}
x(\epsilon) & =\left[1+e^{i(\theta+i \epsilon)} Z A U A^{-1} Q_{1}\left(e^{i(\theta+i \epsilon)}\right)\right] x \\
& \equiv\left[1+L_{+} Q_{1}\left(e^{i \theta_{+}}\right)\right] x
\end{aligned}
$$

\footnotetext{
${ }^{3}$ That the set $M$ has measure zero is a consequence of Lemma
} 
must be bounded in norm as $\epsilon \rightarrow 0$ because we have just seen that if it is unbounded, we have an eigenvalue of the operator $V$. For $y=A^{*} x \in R\left(A^{*}\right)$, the absolutely continuous spectrum, $m_{y}^{a c}$ of $V$ is the limit of

$$
\left\langle\delta_{\epsilon}\left(1-V e^{i \theta}\right) y, y\right\rangle=\left\langle A \delta_{\epsilon}\left(1-V e^{i \theta}\right) A^{*} x, x\right\rangle .
$$

Our aim is to show that this is zero for all $y \in \mathcal{H}$. We define

$$
\begin{aligned}
F_{1}(\theta) & =\left(1-V e^{i \theta}\right)^{-1} \\
F(\theta) & =\left(1-U e^{i \theta}\right)^{-1}
\end{aligned}
$$

and in a similar fashion to (19) and (20), obtain

$$
F_{1}(\theta)=F(\theta)\left[1+(V-U) e^{i \theta} F_{1}(\theta)\right]
$$

and

$$
\left(1+(V-U) e^{i \theta} F_{1}(\theta)\right)=\left(1-(V-U) e^{i \theta} F(\theta)\right)^{-1} .
$$

Writing $X=V-U$, on substituting (27) into our expression for the $\delta$-function (12) we obtain

$$
\begin{aligned}
2 \pi \delta_{\epsilon}\left(1-V e^{i \theta}\right) & =\left(1-e^{-2 \epsilon}\right) F_{1}^{*}\left(\theta_{+}\right) F_{1}\left(\theta_{+}\right) \\
& =\left[1+e^{i \theta_{+}} X F_{1}\left(\theta_{+}\right)\right]^{*} 2 \pi \delta_{\epsilon}\left(1-U e^{i \theta}\right)\left[1+e^{i \theta_{+}} X F_{1}\left(\theta_{+}\right)\right] .
\end{aligned}
$$

Substitution of (14) and noting that

$$
X=V-U=\left(1+A^{*} Z A\right) U-U=A^{*} Z A U
$$

gives us

$$
\begin{aligned}
A & \delta_{\epsilon}\left(1-V e^{i \theta}\right) A^{*} \\
& =A\left[1+X e^{i \theta_{+}} F_{1}\left(\theta_{+}\right)\right]^{*} \delta_{\epsilon}\left(1-U e^{i \theta}\right)\left[1+X e^{i \theta_{+}} F_{1}\left(\theta_{+}\right)\right] A^{*} \\
& =\left[1+L_{+} Q_{1}\left(\theta_{+}\right)\right]^{*} A \delta_{\epsilon}\left(1-U e^{i \theta}\right) A^{*}\left[1+L_{+} Q_{1}\left(\theta_{+}\right)\right] .
\end{aligned}
$$

The absolutely continuous spectrum, $m_{y}^{a c}$ of $V$ is the limit of

$$
\begin{aligned}
& \left\langle A \delta_{\epsilon}\left(1-V e^{i \theta}\right) A^{*} x, x\right\rangle \\
& =\left\langle\left[1+L_{+} Q_{1}\left(\theta_{+}\right)\right]^{*} A \delta_{\epsilon}\left(1-U e^{i \theta}\right) A^{*}\left[1+L_{+} Q_{1}\left(\theta_{+}\right)\right] x, x\right\rangle \\
& =\left\langle A \delta_{\epsilon}\left(1-U e^{i \theta}\right) A^{*} x(\epsilon), x(\epsilon)\right\rangle \\
& =\frac{\epsilon(1-\epsilon)}{\pi}\left\langle G_{\epsilon}(\theta ; U, A) x(\epsilon), x(\epsilon)\right\rangle
\end{aligned}
$$


which tends to zero as $\epsilon \rightarrow 0$ if both $G_{\epsilon}(\theta ; U, A)$ and $x(\epsilon)$ are bounded. $G_{\epsilon}(\theta ; U, A)$ is bounded as we have $\theta \in J \sim N$ and $x(\epsilon)$ is bounded by (24).

Part (ai) follows since $R\left(A^{*}\right)$ cyclic for $U$ implies that $R\left(A^{*}\right)$ is cyclic for $V$.

(b) Let $N_{1}=N(V, A, J)$. We have just shown that $\theta \in J \sim\left(N \cup M \cup \sigma_{p}(V)\right)$ implies that

$$
\frac{\epsilon}{\pi}\left\langle G_{\epsilon}(\theta ; V, A) x(\epsilon), x(\epsilon)\right\rangle \rightarrow 0
$$

and therefore

$$
\left\langle\delta_{\epsilon}\left(1-V e^{i \theta}\right) y, y\right\rangle \rightarrow 0 .
$$

If we can infer the strong limit from this weak limit then we have established that $\theta \notin N_{1}$. We use the result that if $x_{n} \stackrel{w}{\rightarrow} x$ and $\left\|x_{n}\right\| \rightarrow\|x\|$, then $x_{n} \stackrel{s}{\rightarrow} x$ ([23], p 244). Writing $G_{\epsilon}$ and $G$ for $G_{\epsilon}(\theta ; V, A)$ and $G(\theta ; V, A)$, and $F_{\epsilon}$ and $F$ for $F\left(\theta_{+} ; V\right)$ and $F(\theta ; V)$, consider

$$
\begin{aligned}
\left|\left\|G_{\epsilon} x\right\|^{2}-\|G x\|^{2}\right| & \\
& =\left|\left\langle\left(G_{\epsilon}^{2}-G^{2}\right) x, x\right\rangle\right| \\
& =\left|\left\langle A\left\{\left(F_{\epsilon}^{*} F_{\epsilon}-F^{*} F\right) A^{*} A F_{\epsilon}^{*} F_{\epsilon}+F^{*} F A^{*} A\left(F_{\epsilon}^{*} F_{\epsilon}-F^{*} F\right)\right\} A^{*} x, x\right\rangle\right| .
\end{aligned}
$$

If $A, F_{\epsilon}$ and $F$ are bounded operators, then if $F_{\epsilon}^{*} F_{\epsilon}-F^{*} F$ tends to zero as $\epsilon \rightarrow 0$ we can conclude that the strong limit exists. A short calculation shows that

$$
F_{\epsilon}^{*} F_{\epsilon}-F^{*} F=\left[\left(1-e^{-2 \epsilon}\right)-\left(1-e^{-\epsilon}\right)\left(U e^{i \theta}+U^{*} e^{-i \theta}\right)\right] F_{\epsilon}^{*} F_{\epsilon} F^{*} F
$$

which trivially tends to zero as $\epsilon \rightarrow 0$ given the boundedness of $F_{\epsilon}$ and $F$. Finally, $A$ is bounded by assumption and (24) shows that $Q_{1}\left(\theta_{+}\right)$is a bounded operator as $\epsilon \rightarrow 0$ and thus both $F_{\epsilon}$ and $F$ are bounded.

Moving on from (30), we have now established that $N_{1} \subset N \cup M \cup \sigma_{p}(V)$ so $N_{1}$ must have measure zero, again remembering that we need Lemma $\llbracket$ below to prove that $M$ has measure zero. By Theorem 1 .2 $N_{1}$ supports the singular spectrum of $V$. That is,

$$
m^{s}\left(N_{1}^{\mathrm{c}}\right)=0
$$

where the set $N_{1}^{\mathrm{c}}$ is the complement of $N_{1}$. As the measure is positive and $\mathrm{m}^{s}=$ $m^{s c}+m^{p}$, we know that

$$
m^{s c}\left(N_{1}^{\mathrm{c}}\right)=0 .
$$

Trivially, $(N \cup M) \sim \sigma_{p}(V)$ contains $N_{1} \sim \sigma_{p}(V)$. Thus

$$
\begin{aligned}
m^{s c}\left(\left[N_{1} \cap \sigma_{p}(V)^{\mathrm{c}}\right]^{\mathrm{c}}\right) & =m^{s c}\left(N_{1}^{\mathrm{c}} \cup \sigma_{p}(V)\right) \\
& =m^{s c}\left(N_{1}^{\mathrm{c}}\right)+m^{s c}\left(\sigma_{p}(V)\right) \\
& =0+0 \\
& =0
\end{aligned}
$$


as the (continuous) measure of single points is zero.

The set $N \cup M \cap \sigma_{p}(V)^{\mathrm{c}}$ must support $m^{s c}$ as $N_{1} \cap \sigma_{p}(V)^{\mathrm{c}}$ is a subset. Therefore

$$
m^{s c}\left(\left[N \cup M \cap \sigma_{p}(V)^{\mathrm{c}}\right]^{\mathrm{c}}\right)=0 .
$$

This equals

$$
\begin{aligned}
m^{s c}\left([N \cup M]^{c} \cup \sigma_{p}(V)\right) & =m^{s c}\left([N \cup M]^{\mathrm{c}}\right)+m^{s c}\left(\sigma_{p}(V)\right) \\
& =m^{s c}\left([N \cup M]^{\mathrm{c}}\right)
\end{aligned}
$$

so we conclude that the set $N \cup M$ supports the singular continuous part of the spectrum.

Theorem $\llbracket$.4 has shown us that $V$ has an empty absolutely continuous component, and that the singular continuous component is supported by the set $N \cup M$, which is independent of $\lambda$. We know that $N$ has measure zero, and Lemma below shows us that $M$ also has measure zero. This will allow us to apply Theorem 11.6 to show that the singular continuous spectrum of $V$ is also empty. Thus, with both the a.c. and s.c. spectra empty, we can conclude that $V$ must have pure point spectrum.

LEMMA II.5 Let $Q(z)$ be a trace class valued analytic function inside the complex unit circle, with $|z|<1$. Then for a.e. $\theta$

$$
\lim _{\epsilon \downarrow 0} Q\left(e^{i(\theta+i \epsilon)}\right) \equiv Q\left(e^{i(\theta+i 0)}\right)
$$

exists in Hilbert Schmidt norm.

Proof. We parallel the proof of de Branges theorem (see [24] and pages 149150 in [25]). Consider

$$
\begin{aligned}
Q\left(e^{i(\theta+i \epsilon)}\right) & +Q^{*}\left(e^{i(\theta+i \epsilon)}\right) \\
& =\int A^{*}\left\{\frac{1}{1-e^{-i\left(\theta^{\prime}-\theta\right)} e^{-\epsilon}}+\frac{1}{1-e^{i\left(\theta^{\prime}-\theta\right)} e^{-\epsilon}}\right\} A E\left(d \theta^{\prime}\right) \\
& =\int A^{*}\left\{\frac{2\left(1-e^{-\epsilon} \cos \left(\theta^{\prime}-\theta\right)\right)}{1+e^{-2 \epsilon}-2 e^{-\epsilon} \cos \left(\theta^{\prime}-\theta\right)}\right\} A E\left(d \theta^{\prime}\right) .
\end{aligned}
$$

The factor within the curly braces is greater than zero for all $\theta^{\prime}, \theta$ and thus we have

$$
Q\left(e^{i(\theta+i \epsilon)}\right)+Q^{*}\left(e^{i(\theta+i \epsilon)}\right) \geq 0 \forall \epsilon \geq 0 .
$$


Therefore, following de Branges,

$$
\begin{aligned}
\left|\operatorname{det}\left(1+Q\left(e^{i(\theta+i \epsilon)}\right)\right)\right|^{2} & \geq \operatorname{det}\left(1+Q^{*}\left(e^{i(\theta+i \epsilon)}\right) Q\left(e^{i(\theta+i \epsilon)}\right)\right) \\
& =\prod\left(1+\left|\alpha_{n}\right|^{2}\right) \\
& \geq\left\{\begin{array}{l}
\sum\left|\alpha_{n}\right|^{2}=\left\|Q\left(e^{i(\theta+i \epsilon)}\right)\right\|_{H . S .}^{2} \\
1 .
\end{array}\right.
\end{aligned}
$$

$\left\{\alpha_{n}\right\}$ are the eigenvalues of $Q\left(e^{i(\theta+i \epsilon)}\right)$. From the two bounds on $\left|\operatorname{det}\left(1+Q\left(e^{i(\theta+i \epsilon)}\right)\right)\right|$ above, we obtain

$$
\|\left.\frac{Q\left(e^{i(\theta+i \epsilon)}\right)}{\operatorname{det}\left(1+Q\left(e^{i(\theta+i \epsilon)}\right)\right)}\right|_{H . S .} \leq 1 \text { and }\left|\frac{1}{\operatorname{det}\left(1+Q\left(e^{i(\theta+i \epsilon)}\right)\right)}\right| \leq 1 .
$$

The definition of an analytic operator ([9], p. 189) implies the analyticity of the eigenvalues, and thus the operations of taking the determinant and the Hilbert Schmidt norm are analytic. Hence, both functions above are analytic and bounded within the complex unit circle $(\epsilon>0)$. Application of Fatou's theorem ([26], p. 454) establishes the existence in the limit as $\epsilon \rightarrow 0$ and hence both functions exist on the boundary almost everywhere. Taking the quotient we establish the existence of $Q\left(e^{i(\theta+i 0)}\right)$ in the Hilbert Schmidt norm.

Let $(\Omega, \mu)$ be a separable measure space, and

$$
V(\lambda)=\int e^{-i \theta} E_{\lambda}(d \theta)
$$

a measurable family of unitary operators on $\mathcal{H}$. We denote by

$$
\mathbb{V}=\int e^{-i \theta} \mathbb{E}(d \theta)
$$

the multiplication operator

$$
(\mathbb{V} u)(\lambda)=V(\lambda) u(\lambda)
$$

on $L^{2}(\Omega, \mu ; \mathcal{H})$, where $u(\lambda) \in L^{2}(\Omega, \mu ; \mathcal{H})$.

A vector $u(\lambda)$ is an element of $L^{2}(\Omega, \mu ; \mathcal{H})$ if, for $u(\lambda) \in \mathcal{H}$,

$$
\int_{-\infty}^{\infty}\|u(\lambda)\|^{2} d \mu<\infty
$$

It is important to note the difference between $V(\lambda)$ acting on $\mathcal{H}$ and $\mathbb{V}$ acting on $L^{2}(\Omega, \mu ; \mathcal{H})$. To obtain our goal of showing that for a.e. $\lambda, V(\lambda)$ has a pure 
point spectrum, we must show that $\mathbb{V}$ is absolutely continuous as a function of $\lambda$ on the space $L^{2}(\Omega, \mu ; \mathcal{H})$.

Theorem [1.6] is taken directly from [2]. The proof given is, apart from some small notational changes, identical to that in [2]. Due to a number of typographical errors however, we have reproduced the proof here for reference and clarity.

THEOREM II.6 Let $\mathbb{V}$ be absolutely continuous on $L^{2}(\Omega, \mu ; \mathcal{H})$, and assume that there is a fixed set $S$ of Lebesgue measure zero which supports the singular continuous spectrum of $V(\lambda)$ in the interval $J$ for $\mu$-a.e. $\lambda$. Then $V(\lambda)$ has no singular continuous spectrum in $J$ for $\mu$-a.e. $\lambda$.

Proof. For fixed $x \in \mathcal{H}$, and any measurable subset $\Gamma$ of $\Omega$, let $u(\lambda)=\chi_{\Gamma}(\lambda) x$ be a vector in $L^{2}(\Omega, \mu ; \mathcal{H})$. Then

$$
\begin{aligned}
\int_{\Gamma}\left|E_{\lambda}^{s c}[J] x\right|^{2} \mu(d \lambda) & \leq \int_{\Gamma}\left|E_{\lambda}[S] x\right|^{2} \mu(d \lambda) \\
& =\int\left|E_{\lambda}[S] u(\lambda)\right|^{2} \mu(d \lambda) \\
& =\int|\mathbb{E}[S] u(\lambda)|^{2} \mu(d \lambda) \\
& =\|\mathbb{E}[S] u(\lambda)\|^{2}=0 .
\end{aligned}
$$

$\int_{\Gamma}\left|E_{\lambda}^{s c}[J] x\right|^{2} \mu(d \lambda)=0$ implies that $\left|E_{\lambda}^{s c}[J] x\right|^{2}=0$ for $\mu$-a.e. $\lambda$. Thus

$$
E_{\lambda}^{s c}[J] x=0
$$

for every $x \in \mathcal{H}$.

The application of Theorem $\llbracket$ relies on finding a fixed set $S$ of measure zero which supports the singularly continuous spectrum. $S=N \cup M$ is sufficient.

We have now established all the basic requirements for $V$ to be pure point, given $U$ pure point. They are now combined to produce the main theorem of the paper. There is still quite a lot of manipulation to satisfy the condition $\mathbb{V}$ a.c. on $L^{2}(\mathbb{R} ; \mathcal{H})$ of Theorem $\llbracket .6$ and this will be the focus for the remainder of Section $\amalg$ and Section

THEOREM II.7 Let $U$ and A satisfy the hypotheses of Theorem II.4 and define for $\lambda \in \mathbb{R}$

$$
V(\lambda)=e^{i \lambda A^{*} A / \hbar} U .
$$

Then $V(\lambda)$ is pure point in $J$ for a.e. $\lambda$. 
Proof. By Theorem $\llbracket .4$ with $W=\lambda I, V(\lambda)$ has no absolutely continuous spectrum in $J$, and its singularly continuous spectrum is supported on the fixed set $S=N \cup M$. Application of Lemma $\llbracket .5$ shows that $S$ is of measure zero. Theorem 1 .6 applies and shows that the singular continuous spectrum is empty, if we can show that $\mathbb{V}$ is absolutely continuous on $L^{2}(\mathbb{R} ; \mathcal{H})$. We show this in the following sections.

As we have shown that both the absolutely continuous and singular continuous parts of the spectrum are empty, we conclude that $V(\lambda)$ is pure point for a.e. $\lambda \in \mathbb{R}$.

To show that $\mathbb{V}$ is a.c., we apply a modified version of the Putnam-Kato theorem which is proved in Section [III. The unitary Putnam-Kato theorem is

Theorem $\amalg .3$ Let $V$ be unitary, and $D$ a self-adjoint bounded operator. If $C=$ $V\left[V^{*}, D\right] \geq 0$, then $V$ is absolutely continuous on $R\left(C^{1 / 2}\right)$. Hence, if $R\left(C^{1 / 2}\right)$ is cyclic for $V$, then $V$ is absolutely continuous on $\mathcal{H}$.

We apply this theorem on the space $L^{2}(\mathbb{R} ; \mathcal{H})$. A naive application to obtain the desired result is as follows. We slightly change notation and explicitly include the $\lambda$ dependence of $W$ in our definition of $V$. If we choose $\mathbb{D}=-i(d / d \lambda)$, with $V=e^{i \lambda A^{*} W A} U$, we see that

$$
-i \frac{d V^{*}}{d \lambda}=-U^{*} A^{*} W A e^{-i \lambda A^{*} W A}=-V^{*} A^{*} W A
$$

so that for some $u \in L^{2}(\mathbb{R} ; \mathcal{H})$

$$
\begin{aligned}
{\left[\mathbb{V}^{*}, \mathbb{D}\right] u } & =\left(\mathbb{V}^{*} \mathbb{D}-\mathbb{D} \mathbb{V}^{*}\right) u=-\mathbb{D} \mathbb{V}^{*} u \\
& =i \frac{d}{d \lambda}\left(\mathbb{V}^{*} u\right)=\mathbb{V}^{*} A^{*} W A u .
\end{aligned}
$$

Therefore,

$$
\mathbb{C}=\mathbb{V}\left[\mathbb{V}^{*}, \mathbb{D}\right]=A^{*} W A .
$$

With $W=I$, we obtain $\mathbb{C}=A^{*} A \geq 0$ and thus $R\left(\mathbb{C}^{1 / 2}\right)=R\left(A^{*}\right)$ (see the proof to Theorem VI.9 in [9]) is cyclic for $V$. Hence, $\mathbb{V}$ is a.c. and we satisfy all the requirements of Theorem

The problem here is that $\mathbb{D}$ is not bounded, and boundedness of $\mathbb{D}$ is essential in the proof of the Putnam-Kato theorem. We use a similar technique as Howland [2] to overcome this issue.

As the norm of $A^{*} A$ may be scaled however we like, we can rewrite $V$, for real $t$ as

$$
V(t)=e^{i c t A^{*} A} U
$$

for some real $c>0$. 
Proposition II.8 On $L^{2}(\mathbb{R} ; \mathcal{H})$, consider the unitary multiplication operator $\mathbb{V}$, defined by

$$
\mathbb{V} u(t)=V(t) u(t)=e^{i c t A^{*} A} U u(t)
$$

and the bounded self-adjoint operator $\mathbb{D}=-\arctan (p / 2)$, where $p=-i d / d t$. Then $C=\mathbb{V}\left[\mathbb{V}^{*}, D\right]$ is positive definite, and $R\left(C^{1 / 2}\right)$ is cyclic for $\mathbb{V}$. Hence, the requirements of Theorem 1 II.7 are fully satisfied.

Proof. The operator $\mathbb{D}$ on $L^{2}(\mathbb{R} ; \mathcal{H})$ is convolution by the Fourier transform of $-\arctan (x / 2)$ [2], which is $i \pi t^{-1} e^{-2|t|}([27]$, p. 87, (3)). This is a singular (principal value) integral operator, because $\arctan (p / 2)$ does not vanish at infinity. Thus, for $u(t) \in L^{2}(\mathbb{R} ; \mathcal{H})$,

$$
\mathbb{D} u(t)=i \pi P \int_{-\infty}^{\infty} \frac{e^{-2|t-y|}}{t-y} u(y) d y
$$

and

$$
\left[\mathbb{V}^{*}, \mathbb{D}\right] u(t)=i \pi P \int_{-\infty}^{\infty} e^{-2|t-y|} \frac{V^{*}(t)-V^{*}(y)}{t-y} u(y) d y
$$

So

$$
\begin{aligned}
\mathbb{C} u(t) & =\mathbb{V}\left[\mathbb{V}^{*}, \mathbb{D}\right] u(t) \\
& =i \pi P \int_{-\infty}^{\infty} e^{-2|t-y|} \frac{1-V(t) V^{*}(y)}{t-y} u(y) d y .
\end{aligned}
$$

Inserting expression (31) for $V(t)$, we obtain

$$
\begin{aligned}
\mathbb{C} u(t) & =i \pi \int_{-\infty}^{\infty} e^{-2|t-y|} \frac{1-e^{i c(t-y) A^{*} A}}{t-y} u(y) d y \\
& =i \pi \int_{-\infty}^{\infty} e^{-2|t-y|} \frac{1-\cos \left(A^{*} A c(t-y)\right)-i \sin \left(A^{*} A c(t-y)\right)}{t-y} u(y) d y
\end{aligned}
$$

Note that this is no longer a singular integral. To show that $\mathbb{C}$ is positive, we must show that

$$
(u(t), \mathbb{C} u(t))>0 \quad \forall u(t) \in L^{2}(\mathbb{R} ; \mathcal{H}) .
$$

Note that the inner product on $L^{2}(\mathbb{R} ; \mathcal{H})$ is given by

$$
\left(u(t), u^{\prime}(t)\right)=\int_{-\infty}^{\infty} u^{*}(t) u^{\prime}(t) d t .
$$

We now write our operator $A$ in terms of its spectral components. Note that here $\lambda$ decomposes $A$ and bears no relation to the strength parameter used at other stages 
in this paper. When required for clarity, we write $\int_{\lambda}$ to identify the integral over the variable $\lambda$.

$$
A=\int \lambda E(d \lambda)
$$

A general vector $u(t)$ may be written

$$
u(t)=\int E(d \lambda) u(t)
$$

Then

$$
f(A) u(t)=\int f(\lambda) E(d \lambda) u(t)
$$

which implies that we may rewrite (33) as

$$
\begin{aligned}
\mathbb{C} u(t) & =i \pi \int_{-\infty}^{\infty} d y \int_{\lambda} e^{-2|t-y|} \frac{1-e^{i c(t-y)|\lambda|^{2}}}{t-y} E(d \lambda) u(y) \\
& =\int_{-\infty}^{\infty} d y \int_{\lambda} \phi_{\lambda}(t-y) E(d \lambda) u(y) \\
& =\int_{\lambda} E(d \lambda) \mathcal{C}_{\lambda}(t)
\end{aligned}
$$

where

$$
\mathcal{C}_{\lambda}(t)=\int_{-\infty}^{\infty} d y \phi_{\lambda}(t-y) u(y)
$$

and we have defined the new function

$$
\phi_{\lambda}(t)=i \pi e^{-2|t|} t^{-1}\left(1-e^{i c t|\lambda|^{2}}\right) .
$$

By the convolution theorem, note that

$$
\tilde{\mathcal{C}}_{\lambda}(\omega)=\tilde{\phi}_{\lambda}(\omega) \tilde{u}(\omega)
$$

where the " $\sim$ " indicates Fourier transform.

Using this decomposition of $u(t)$ and Parseval's theorem, we can now easily write down $(u(t), \mathbb{C} u(t))$. We use $(x, y)_{\mathcal{H}}$ to indicate the inner product on the Hilbert Space $\mathcal{H}$, reserving $(x, y)$ for the inner product on $L^{2}(\mathbb{R} ; \mathcal{H})$ as in (34).

$$
\begin{aligned}
(u(t), \mathbb{C} u(t)) & =\int_{-\infty}^{\infty} d t(u(t), \mathbb{C} u(t))_{\mathcal{H}} \\
& =\int_{-\infty}^{\infty} d t\left(u(t), \int_{\lambda} E(d \lambda) \mathcal{C}_{\lambda}(t)\right)_{\mathcal{H}} \\
& =\int_{-\infty}^{\infty} d t\left(u(t), \int_{\lambda} E(d \lambda) \int \frac{d \omega}{2 \pi} e^{i \omega t} \tilde{\mathcal{C}}_{\lambda}(\omega)\right)_{\mathcal{H}} \\
& =\int_{\lambda} E(d \lambda) \int \frac{d \omega}{2 \pi}\left|\tilde{u}_{\lambda}(\omega)\right|^{2} \tilde{\phi}_{\lambda}(\omega) .
\end{aligned}
$$


We clearly see that if $\tilde{\phi}_{\lambda}(\omega)$ is positive for all $\lambda$ then $\mathbb{C}$ will be positive.

In the following calculation we will find the need to bound $c|\lambda|^{2}$. The restriction $0 \leq c|\lambda|^{2} \leq 1$ will be employed. We argue that as $A^{*} A$ is a positive self-adjoint bounded operator we can restrict the integral over $\lambda$ to ([28], p. 262, 273)

$$
A^{*} A=\int_{-\infty}^{\infty}|\lambda|^{2} E(d \lambda)=\int_{m-0}^{M}|\lambda|^{2} E(d \lambda)
$$

where $M$ is the least upper bound and $m$ the greatest lower bound of $A^{*} A$. The norm of $A^{*} A$ is given by $\max (|m|,|M|)$. Thus, if we set

$$
c=\frac{1}{\left\|A^{*} A\right\|}
$$

then we guarantee each $c|\lambda|^{2}$ to be less than unity.

Proceeding, the Fourier transform, $\tilde{\phi}_{\lambda}(\omega)$ of

$$
\phi_{\lambda}(t)=i \pi e^{-2|t|} t^{-1}\left[1-\cos c t|\lambda|^{2}-i \sin c t|\lambda|^{2}\right]
$$

is now calculated. We split 36 into two parts.

$$
\begin{aligned}
& \phi_{\lambda 1}(t)=i \pi e^{-2|t|} t^{-1}\left[1-\cos c t|\lambda|^{2}\right] \\
& \phi_{\lambda 2}(t)=\pi e^{-2|t|} t^{-1} \sin c t|\lambda|^{2} .
\end{aligned}
$$

The Fourier transform of (37) is

$$
\begin{aligned}
\tilde{\phi}_{\lambda 1}(\omega)=i \pi & \int_{-\infty}^{\infty} e^{-2|t|} t^{-1}\left(1-\cos c t|\lambda|^{2}\right) e^{-i \omega t} d t \\
=i \pi & {\left[\int_{0}^{\infty} e^{-2 t} t^{-1}\left(1-\cos c t|\lambda|^{2}\right) e^{-i \omega t} d t\right.} \\
& \left.+\int_{0}^{\infty} e^{-2 t}\left(-t^{-1}\right)\left(1-\cos c t|\lambda|^{2}\right) e^{i \omega t} d t\right] .
\end{aligned}
$$

Using ([27], p. 157, (59)), and setting $S=c|\lambda|^{2} /(2+i \omega)$, we obtain

$$
\tilde{\phi}_{\lambda 1}(\omega)=\frac{i \pi}{2} \log \left(\frac{1+S^{2}}{1+S^{* 2}}\right) .
$$

The logarithm of a complex number can in general be written as

$$
\log (z)=\log (|z|)+i \operatorname{Arg} z
$$


so noting that $\left|\left(1+S^{2}\right) /\left(1+S^{* 2}\right)\right|=1$, we see that

$$
\begin{aligned}
\tilde{\phi}_{\lambda 1}(\omega) & =-\frac{\pi}{2} \operatorname{Arg}\left(\frac{1+S^{2}}{1+S^{* 2}}\right) \\
& =-\pi \operatorname{Arg}\left(1+S^{2}\right) .
\end{aligned}
$$

With $\kappa=c|\lambda|^{2}$, the real and imaginary parts of $1+S^{2}$ are

$$
\begin{aligned}
& \Re\left(1+S^{2}\right)=\frac{\left(4+\omega^{2}\right)^{2}+\kappa^{2}\left(4-\omega^{2}\right)}{\left(4+\omega^{2}\right)^{2}} \\
& \Im\left(1+S^{2}\right)=\frac{-4 \kappa^{2} \omega}{\left(4+\omega^{2}\right)^{2}} .
\end{aligned}
$$

With the restriction that $0 \leq \kappa \leq 1$, the real part is positive for all $\omega$ and thus $\operatorname{Arg}(z)=\arctan (\Im z / \Re z)$. Thus,

$$
\tilde{\phi}_{\lambda 1}(\omega)=-\pi \arctan \left(\frac{\Im\left(1+S^{2}\right)}{\Re\left(1+S^{2}\right)}\right) .
$$

$\arctan (z)$ is the principal part of $\operatorname{Arctan}(z)$, with range $-\pi / 2<\arctan (z)<$ $\pi / 2$. The Fourier transform of (38) is similarly calculated using ([27], p. 152, (16)), to be

$$
\begin{aligned}
\tilde{\phi}_{\lambda 2}(\omega) & =\pi\left[\arctan S+\arctan S^{*}\right] \\
& =\pi\left[\arctan \left(\frac{c|\lambda|^{2}}{2+i \omega}\right)+\arctan \left(\frac{c|\lambda|^{2}}{2-i \omega}\right)\right] .
\end{aligned}
$$

Repeated application of the formula $\arctan \left(z_{1}\right)+\arctan \left(z_{2}\right)=\arctan \left(z_{1}+z_{2} / 1-\right.$ $z_{1} z_{2}$ ), valid when $z_{1} z_{2}<1$ (true for $0 \leq \kappa \leq 1$ ), yields $^{4}$

$$
\begin{aligned}
\tilde{\phi}_{\lambda}(\omega) & =\tilde{\phi}_{\lambda 1}(\omega)+\tilde{\phi}_{\lambda 2}(\omega) \\
& =\pi \arctan \left(\frac{n\left(\omega, c|\lambda|^{2}\right)}{d\left(\omega, c|\lambda|^{2}\right)}\right)
\end{aligned}
$$

where

$$
n(\omega, \kappa)=4 \kappa\left[\left(4+\omega^{2}\right)^{2}+\kappa \omega\left(4+\omega^{2}\right)+\kappa^{2}\left(4-\omega^{2}\right)-\kappa^{3} \omega\right]
$$

and

$$
d(\omega, \kappa)=\left(4+\omega^{2}\right)^{3}-2 \kappa^{2} \omega^{2}\left(4+\omega^{2}\right)-16 \kappa^{3} \omega-\kappa^{4}\left(4-\omega^{2}\right) .
$$

\footnotetext{
${ }^{4}$ This result is not valid for values of $\kappa$ larger than around 2 , at which point the arctan addition formulas fail - this is a moot point however, as we may trivially restrict $\kappa$ as already explained.
} 
One may easily confirm that for $0 \leq \kappa \leq 1, n(\omega, \kappa) / d(\omega, \kappa)$ and hence $\tilde{\phi}_{\lambda}(\omega)$ is strictly positive by noting that there are four distinct regions of interest for $\omega$, in which terms in $n$ and $d$ do not change sign. Table 1 shows these regions and the sign of each term in the region. Note that the global (positive and hence irrelevant) $\kappa$ factor from (40) is dropped from the numerator for the following discussion.

\begin{tabular}{|c|c|c|c|c|}
\hline$n(\omega, \kappa)=$ & $\left(4+\omega^{2}\right)^{2}$ & $+\kappa \omega\left(4+\omega^{2}\right)^{2}$ & $+\kappa^{2}\left(4-\omega^{2}\right)$ & $-\kappa^{3} \omega$ \\
\hline$\omega<-2$ & $+\mathrm{ve}$ & $-\mathrm{ve}$ & $-\mathrm{ve}$ & $+\mathrm{ve}$ \\
$-2<\omega<0$ & $+\mathrm{ve}$ & $-\mathrm{ve}$ & $+\mathrm{ve}$ & $+\mathrm{ve}$ \\
$0<\omega<2$ & $+\mathrm{ve}$ & $+\mathrm{ve}$ & $+\mathrm{ve}$ & $-\mathrm{ve}$ \\
$\omega>2$ & $+\mathrm{ve}$ & $+\mathrm{ve}$ & $-\mathrm{ve}$ & $-\mathrm{ve}$ \\
\hline \hline$d(\omega, \kappa)=$ & $\left(4+\omega^{2}\right)^{3}$ & $-2 \kappa^{2} \omega^{2}\left(4+\omega^{2}\right)$ & $-16 \kappa^{3} \omega$ & $-\kappa^{4}\left(4-\omega^{2}\right)$ \\
\hline$\omega<-2$ & $+\mathrm{ve}$ & $-\mathrm{ve}$ & $+\mathrm{ve}$ & $+\mathrm{ve}$ \\
$-2<\omega<0$ & $+\mathrm{ve}$ & $-\mathrm{ve}$ & $+\mathrm{ve}$ & $-\mathrm{ve}$ \\
$0<\omega<2$ & $+\mathrm{ve}$ & $-\mathrm{ve}$ & $-\mathrm{ve}$ & $-\mathrm{ve}$ \\
$\omega>2$ & $+\mathrm{ve}$ & $-\mathrm{ve}$ & -ve & $+\mathrm{ve}$ \\
\hline
\end{tabular}

Table 1: Sign of each term in the numerator $n(\omega, \kappa)$ and the denominator $d(\omega, \kappa)$ of (39).

For each row in the table, we simply need to show that the terms add to produce a strictly positive number. First note that the first column for both the numerator and denominator is independent of $\kappa$. To show the positivity of each row, we set all positive $\kappa$-dependent terms to zero and then take $\kappa=1$ for the negative terms to maximise their contribution. Expanding out terms, it is then trivially seen in all cases that the first column $\left(\left(4+\omega^{2}\right)^{2}\right.$ for the numerator and $\left(4+\omega^{2}\right)^{3}$ for the denominator) dominates. Thus, no row is negative and we conclude that $\tilde{\phi}_{\lambda}$ is positive definite.

We have established that the Fourier transform of $\phi_{\lambda}$ is positive definite for $c|\lambda|^{2} \leq 1$. As a visual aid, Figure 1 shows $\tilde{\phi}_{\lambda}(\omega)$. The positivity for $c|\lambda|^{2} \leq 1$ is clear. 


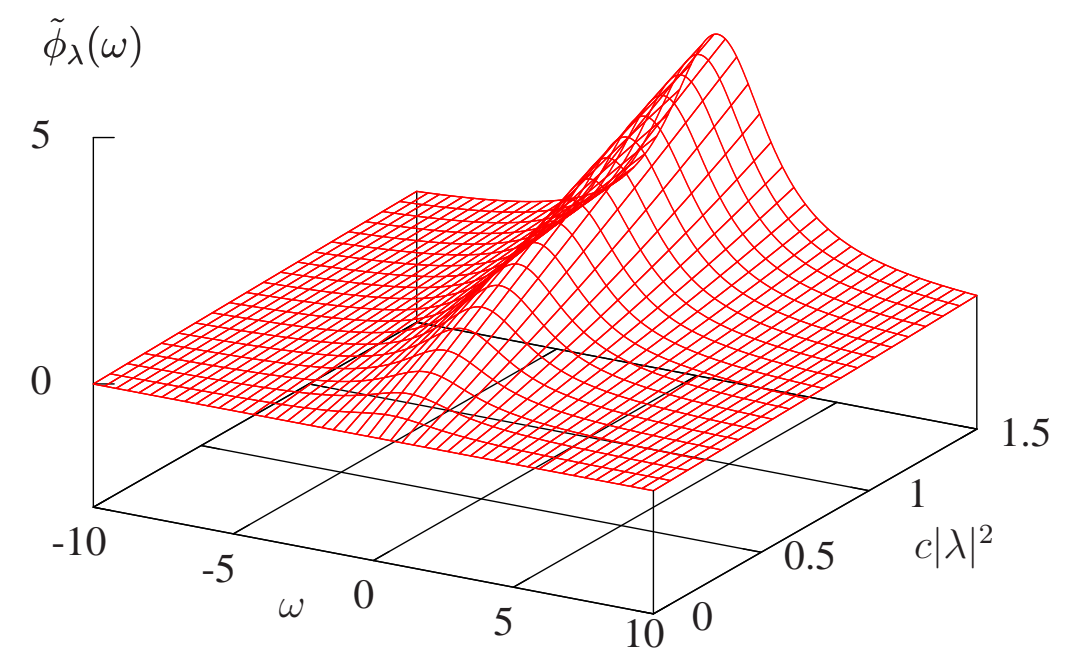

Figure 1: Plot of $\tilde{\phi}_{\lambda}(\omega)=\tilde{\phi}_{\lambda 1}(\omega)+\tilde{\phi}_{\lambda 2}(\omega)$, the Fourier transform of $\phi_{\lambda}(t)=$ $i \pi e^{-2|t|} t^{-1}\left(1-\cos \left(c t|\lambda|^{2}\right)-i \sin \left(c t|\lambda|^{2}\right)\right) . \quad \tilde{\phi}_{\lambda}(\omega)$ is strictly positive for all $\omega$ when $c|\lambda|^{2} \leq 1$.

Thus, $\mathbb{C}$ is strictly positive and $\mathbb{V}$ is absolutely continuous on $R\left(\mathbb{C}^{1 / 2}\right)$. As $A^{*} A$ is a factor of $1-e^{i c t A^{*} A}$ (i.e., $A^{*} A$ is a factor of $\mathbb{C}$ ), $R\left(\mathbb{C}^{1 / 2}\right)=R\left(A^{*}\right)$. Noting that $R\left(A^{*}\right)$ is cyclic for $U$ and hence cyclic for $V$, we conclude that $R\left(\mathbb{C}^{1 / 2}\right)$ is cyclic for $\mathbb{V}$. Thus, $\mathbb{V}$ is absolutely continuous on $L^{2}(\mathbb{R} ; \mathcal{H})$.

We have now satisfied all the requirements of Theorem $\llbracket .7$

\section{The unitary Putnam-Kato theorem}

In this section, we will prove a modified version of the Putnam-Kato theorem, as used in the preceding pages. The theorems and proofs follow a similar argument to that of Reed and Simon ([17], p. 157, Theorem XIII.28) and are motivated by the stroboscopic nature of the kicked Hamiltonian.

DEFINITION III.1 (V-SMOOTH) Let $V$ be a unitary operator. $A$ is $V$-smooth if and only if for all $\phi \in \mathcal{H}, V(t) \phi \in D(A)$ for almost every $t \in \mathbb{R}$ and for some 
constant $C$,

$$
\sum_{n}\left\|A V^{n} \phi\right\|^{2} \leq C\|\phi\|^{2} .
$$

THEOREM III.2 If $A$ is $V$-smooth, then $\overline{R\left(A^{*}\right)} \subset \mathcal{H}_{a c}(V)$.

Proof. Since $\mathcal{H}_{a c}(V)$ is closed, we need only show $R\left(A^{*}\right) \subset \mathcal{H}_{a c}(V)$. Let $\phi \in D\left(A^{*}\right), \psi=A^{*} \phi$, and let $d \mu_{\psi}$ be the spectral measure for $V$ associated with $\psi$. Define, for the period $T$ in (11)

$$
\mathcal{F}_{n}(T)=\frac{1}{\sqrt{2 \pi}}\left(A^{*} \phi,[V(T)]^{n} \psi\right) .
$$

We calculate, droppoing the $T$ for clarity,

$$
\begin{aligned}
\left|\mathcal{F}_{n}\right| & =\frac{1}{\sqrt{2 \pi}}\left|\left(\phi, A V^{n} \psi\right)\right| \\
& \leq \frac{1}{\sqrt{2 \pi}}\|\phi\|\left\|A V^{n} \psi\right\| .
\end{aligned}
$$

Because $A$ is $V$-smooth, we see that

$$
\begin{aligned}
\sum_{n}\left|\mathcal{F}_{n}\right|^{2} & \leq \frac{1}{2 \pi}\|\phi\|^{2} \sum_{n}\left\|A V^{n} \psi\right\|^{2} \\
& \leq \frac{C}{2 \pi}\|\phi\|^{2}\|\psi\|^{2} \\
& <\infty .
\end{aligned}
$$

So $\mathcal{F}_{n} \in L^{2}(\mathbb{R})$. By the Riesz-Fischer theorem ([29], p. 96-7, 4.26 Fourier Series), $\mathcal{F}(\theta)=\frac{1}{\sqrt{2 \pi}} \sum_{n} \mathcal{F}_{n} e^{-i n \theta} \in L^{2}$.

The spectral resolution of $V[T]$ is

$$
V[T]=\int_{0}^{2 \pi} e^{i \theta} d E_{T}(\theta)
$$

so we have

$$
(V[T])^{n}=\int_{0}^{2 \pi} e^{i n \theta} d E_{T}(\theta) .
$$

Therefore, from (42) we obtain

$$
\begin{aligned}
\mathcal{F}_{n} & =\frac{1}{\sqrt{2 \pi}} \int_{0}^{2 \pi}\left(A^{*} \phi, e^{i n \theta} d E_{T}(\theta) \psi\right) \\
& =\frac{1}{\sqrt{2 \pi}} \int_{0}^{2 \pi} e^{i n \theta}\left(\psi, d E_{T}(\theta) \psi\right) \\
& =\frac{1}{\sqrt{2 \pi}} \int_{0}^{2 \pi} e^{i n \theta} d \mu_{\psi}(\theta) .
\end{aligned}
$$


Using the inverse of the expression above for $\mathcal{F}(\theta)$ gives

$$
\mathcal{F}_{n}=\frac{1}{\sqrt{2 \pi}} \int_{0}^{2 \pi} e^{i n \theta} \mathcal{F}(\theta) d \theta
$$

As we have just shown that $\mathcal{F}(\theta) \in L^{2}, d \mu_{\psi}(\theta)=\mathcal{F}(\theta) d \theta$ is absolutely continuous, which implies that $\psi \in R\left(A^{*}\right)$ is in $\mathcal{H}_{a c}(V)$ and so $\overline{R\left(A^{*}\right)} \subset \mathcal{H}_{a c}(V)$.

TheOREM III.3 (UNitARY PUTNAM-Kato THEOREM) Let $V$ be a unitary operator, and $A$ a self-adjoint bounded operator. If $C=V\left[V^{*}, A\right] \geq 0$, then $V$ is absolutely continuous on $R\left(C^{1 / 2}\right)$. Hence, if $R\left(C^{1 / 2}\right)$ is cyclic for $V$, then $V$ is absolutely continuous.

Proof. The discrete time-evolution of an operator $A$ is given by

$$
\mathcal{F}_{n}=V^{-n} A V^{n} \text {. }
$$

We calculate

$$
\begin{aligned}
\mathcal{F}_{n}-\mathcal{F}_{n-1} & =V^{-n} V\left[V^{*}, A\right] V^{n} \\
& \equiv G_{n}
\end{aligned}
$$

so

$$
\begin{aligned}
\sum_{n=a}^{b}\left(\phi, G_{n} \phi\right) & =\sum_{n=a}^{b}\left(\phi, V^{-n} V\left[V^{*}, A\right] V^{n} \phi\right) \\
& =\sum_{n=a}^{b}\left(V^{n} \phi, V\left[V^{*}, A\right] V^{n} \phi\right) \\
& =\sum_{n=a}^{b}\left(C^{\frac{1}{2}} V^{n} \phi, C^{\frac{1}{2}} V^{n} \phi\right) \\
& =\sum_{n=a}^{b}\left\|C^{\frac{1}{2}} V^{n} \phi\right\|^{2}
\end{aligned}
$$

where $C=V\left[V^{*}, A\right]$. We also have

$$
\sum_{n=a}^{b}\left(\phi, G_{n} \phi\right)=\left(\phi, V^{-b} A V^{b} \phi\right)-\left(\phi, V^{-(a-1)} A V^{(a-1)} \phi\right) .
$$


Taking the modulus and using the Schwartz inequality, we obtain

$$
\begin{aligned}
\sum_{n=a}^{b}\left\|C^{\frac{1}{2}} V^{n} \phi\right\|^{2} & \leq 2\left|\left(\phi, V^{-b} A V^{b} \phi\right)\right| \\
& =2\left|\left(V^{b} \phi, A V^{b} \phi\right)\right| \\
& \leq 2\|A\|\left\|V^{b} \phi\right\|^{2} \\
& =2\|A\|\|\phi\|^{2} \\
& <\infty
\end{aligned}
$$

and thus we see that $C^{1 / 2}$ is $V$-smooth.

That $V$ is absolutely continuous on $R\left(C^{1 / 2}\right)$ follows directly from Theorem

\section{Finite rank perturbations}

Here, we utilise the results of Section 1 to show that perturbations of the form (5) lead to the Floquet operator having pure point spectrum for a.e. perturbation strength $\lambda$.

We use directly the definition of strongly $H$-finite from Howland.

Definition IV.1 (STRONGLy H-Finite) Let $H$ be a self-adjoint operator on $\mathcal{H}$ with pure point spectrum, $\phi_{n}$ a complete orthonormal set of eigenvectors, and $H \phi_{n}=\alpha_{n} \phi_{n}$. A bounded operator $A: \mathcal{H} \rightarrow \mathcal{K}$ is strongly $H$-finite if and only if

$$
\sum_{n=1}^{\infty}\left|A \phi_{n}\right|<\infty .
$$

If $H$ is thought of as a diagonal matrix on $l_{2}$, i.e., $H=\sum_{n} \alpha_{n}\left|\phi_{n}\right\rangle\left\langle\phi_{n}\right|$, and $A$ as an infinite matrix $\left\{a_{i j}\right\}$, i.e., $A=\sum_{m, n} a_{m n}\left|\phi_{m}\right\rangle\left\langle\phi_{n}\right|$, then (43) says

$$
\sum_{n}\left[\sum_{i}\left|a_{i n}\right|^{2}\right]^{\frac{1}{2}}<\infty .
$$

For our purposes, we need to show that if $A$ is strongly $H$-finite, then it is $U$ finite. To satisfy the assumption that $Q_{\epsilon}$ is trace class in Lemma $\llbracket .5$ (and hence also compact in Theorem $\llbracket$ (I.4) we also need to show that $A$ is trace class.

THEOREM IV.2 If $A$ is strongly $H$-finite, then given $U=e^{i T H / \hbar}$ for the period $T$ in (1) and $H \phi_{n}=\alpha_{n} \phi_{n}$, 
(a) A is trace class, and

(b) A is U-finite.

Proof. (a) Simply consider

$$
\operatorname{tr}(A)=\sum_{l}\left\langle\phi_{l}|A| \phi_{l}\right\rangle=\sum_{l} a_{l l} \leq \sum_{l}\left|a_{l l}\right| .
$$

For each term in the sum (45) we trivially have

$$
\left|a_{l l}\right| \leq \sqrt{\sum_{i}\left|a_{i l}\right|^{2}}
$$

and thus (45) is finite so $A$ is trace class.

(b) Noting that

$$
U\left|\phi_{n}\right\rangle=e^{i T H / \hbar}\left|\phi_{n}\right\rangle
$$

we calculate, by insertion of a complete set of states,

$$
\begin{aligned}
\sum_{n} & \left\langle\phi_{n}\left|G_{\epsilon}(\theta ; U, A)\right| \phi_{n}\right\rangle \\
& =\sum_{n}\left\langle\phi_{n}\left|A \frac{1}{\left(1-U^{*} e^{-i \theta_{-}}\right)\left(1-U e^{i \theta_{+}}\right)} A^{*}\right| \phi_{n}\right\rangle \\
& =\sum_{m} \frac{\left\langle\phi_{m}\left|A^{*} A\right| \phi_{m}\right\rangle}{\left|1-e^{-\epsilon} e^{i T \alpha_{m} / \hbar} e^{i \theta}\right|^{2}} .
\end{aligned}
$$

The trace norm is then

$$
\operatorname{tr} G_{\epsilon}(\theta)=\sum_{n} \frac{\left|A \phi_{n}\right|^{2}}{\left|1-e^{-\epsilon} e^{i T \alpha_{n} / \hbar} e^{i \theta}\right|^{2}} .
$$

If this is bounded for $\epsilon=0$, then it is trivially bounded for all $\epsilon>0$. By (43) and a slightly modified version of Theorem 3.1 in [2] this is finite a.e. for $\epsilon=0$. Thus the trace norm of $G_{\epsilon}$ exists as $\epsilon \rightarrow 0$, which implies that the strong limit of $G_{\epsilon}$ exists and we conclude that $A$ is $U$-finite.

THEOREM IV.3 Let $U$ be a pure point unitary operator, and let $A_{1}, \ldots, A_{N}$ be strongly $H$-finite. Assume that the $A_{k}$ s commute with each other. Then for a.e. $\lambda=\left(\lambda_{1}, \ldots, \lambda_{N}\right)$ in $\mathbb{R}^{N}$,

$$
V(\lambda)=e^{i\left(\sum_{k=1}^{N} \lambda_{k} A_{k}^{*} A_{k}\right) / \hbar} U
$$

is pure point. 
Proof. This is a trivial modification of Theorem 4.3 in [2]. Let

$$
\mathcal{K}=\bigoplus_{k=1}^{N} \bar{R}\left(A_{k}\right)
$$

The elements of $\mathcal{K}$ are represented as column vectors. Our operator $A: \mathcal{H} \rightarrow \mathcal{K}$ is defined, for $y \in \mathcal{H}$, by

$$
A y=\left[\begin{array}{c}
A_{1} y \\
\vdots \\
A_{N} y
\end{array}\right]=\left[\begin{array}{c}
x_{1} \\
\vdots \\
x_{N}
\end{array}\right]
$$

and therefore $A^{*}: \mathcal{K} \rightarrow \mathcal{H}$ is given by

$$
A^{*} x=A_{1}^{*} x_{1}+\cdots+A_{N}^{*} x_{N}
$$

Accordingly, we introduce $G_{\epsilon}(\theta): \mathcal{K} \rightarrow \mathcal{K}$, the matrix equivalent of equation (9)

$$
\begin{aligned}
& G_{\epsilon}(\theta ; U, A) \\
& \quad=A\left[1-U^{*} e^{-i \theta_{-}}\right]^{-1}\left[1-U e^{i \theta_{+}}\right]^{-1} A^{*} \\
& \quad=\left\{A_{i}\left[1-U^{*} e^{-i \theta_{-}}\right]^{-1}\left[1-U e^{i \theta_{+}}\right]^{-1} A_{j}^{*}\right\}_{1 \leq i, j \leq N^{*}}
\end{aligned}
$$

The diagonal terms are finite a.e. because each $A_{k}$ is $U$-finite by Theorem IV.2 The off diagonal terms are of the form $X_{1}^{*} X_{2}$, and so the Schwartz inequality,

$$
\left|X_{1}^{*} X_{2}\right|^{2} \leq\left\|X_{1}\right\|^{2}\left\|X_{2}\right\|^{2}
$$

ensures that they are finite a.e. too. Hence, $A$ is $U$-finite as every term in the matrix $G_{\epsilon}(\theta ; U, A)$ is a.e. finite as $\epsilon \rightarrow 0$.

Our Hamiltonian may now be written as

$$
H(\lambda)=H_{0}+A^{*} W(\lambda) A \sum_{n=0}^{\infty} \delta(t-n T)
$$

and our Floquet operator as

$$
V(\lambda)=e^{i A^{*} W(\lambda) A / \hbar} U
$$

where $W(\lambda)=\operatorname{diag}\left\{\lambda_{k}\right\}$. In this form, the formalism of Section is essentially

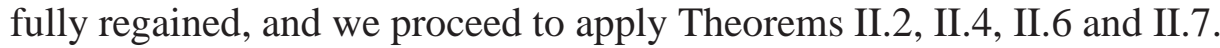

To establish the absolute continuity of the multiplication operator $\mathbb{V}$ on the space $L^{2}\left(\mathbb{R}^{N} ; \mathcal{H}\right)$ we proceed as in Proposition $\llbracket .8$ 
We write

$$
V\left(t_{1}, \ldots, t_{N}\right)=e^{i c \sum_{k=1}^{N} t_{k} A_{k}^{*} A_{k} / \hbar} U
$$

define

$$
D=-\sum_{k=1}^{N} \arctan \left(p_{k} / 2\right)
$$

where $p_{k}=-i d / d t_{k}$, and compute

$$
C=\mathbb{V}\left[\mathbb{V}^{*}, D\right]=\sum_{k=1}^{N} C_{k} \geq 0 .
$$

In obtaining $C$ as a direct sum of the $C_{k}$, we have had to assume that the $A_{k} \mathrm{~s}$ commute with each other. This complication comes when considering the term

$$
V\left(t_{1}, \ldots, t_{N}\right) V^{*}\left(t_{1}, \ldots, t_{k-1}, y_{k}, t_{k+1}, \ldots, t_{N}\right)
$$

in the equivalent of (32). To obtain the required form of $e^{i c\left(t_{k}-y_{k}\right) A_{k}^{*} A_{k}}$ we need the $A_{k}$ s to commute .

Moving on, each $C_{k} \geq 0$ is equivalent to $C$ in Proposition $\llbracket .8$ and hence positive. Finally, we must show that $R\left(C^{1 / 2}\right)$ is cyclic for $\mathbb{V}$. This is no longer trivial as, for each $k$, while we have $R\left(C_{k}^{1 / 2}\right)=R\left(A_{k}^{*}\right)$, the range of $A_{k}^{*}$ is not cyclic for $U$, hence $\mathbb{V}$. To proceed, first note that

$$
R\left(A^{*}\right)=\bigcup_{k} R\left(A_{k}^{*}\right)
$$

Now, as argued in Howland, we can assume that $R\left(A^{*}\right)$ is cyclic for $U$. To elaborate, define $\mathcal{M}\left(U, R\left(A^{*}\right)\right)$ to be the smallest closed reducing subspace of $\mathcal{H}$ containing $R\left(A^{*}\right)$. If $R\left(A^{*}\right)$ is not cyclic for $U$, then $\mathcal{H} \ominus \mathcal{M}$ is not empty. However, as shown below, if $y \in \mathcal{H} \ominus \mathcal{M}$, then $A^{*} W A y=0$, so in $\mathcal{H} \ominus \mathcal{M}, V(t)=U$ and is therefore pure point trivially. Thus, we can ignore the space $\mathcal{H} \ominus \mathcal{M}$, and restrict our discussion to $\mathcal{M}$-i.e., we may assume $R\left(A^{*}\right)$ cyclic for $U$.

The above relied upon showing that $A^{*} W A y=0$ for $y \in \mathcal{H} \ominus \mathcal{M}$. We now prove this. If $y \in \mathcal{H} \ominus \mathcal{M}$ and $y^{\prime} \in \mathcal{M}$, then

$$
\left\langle y, y^{\prime}\right\rangle=0
$$

Given $y^{\prime} \in \mathcal{M}$, there exists an $x \in \mathcal{K}$ such that $y^{\prime}=A^{*} x$, so

$$
\left\langle y, A^{*} x\right\rangle=0 .
$$

\footnotetext{
${ }^{5}$ This restriction is not required in Howland's self-adjoint work because the summation over $k$ in the Hamiltonian (46) enters directly, rather than in the exponent of $V$.
} 
That is

$$
\langle A y, x\rangle=0 .
$$

This is true for all $x \in \mathcal{K}$. Suppose $y^{\prime \prime} \in \mathcal{H}$. Then $W A y^{\prime \prime} \in \mathcal{K}$ and so

$$
\left\langle A y, W A y^{\prime \prime}\right\rangle=0 .
$$

That is

$$
\left\langle A^{*} W A y, y^{\prime \prime}\right\rangle=0 \text {. }
$$

As this is true for any $y^{\prime \prime} \in \mathcal{H}$, we conclude that $A^{*} W A y=0$ on $\mathcal{H} \ominus \mathcal{M}$.

Thus, $R\left(A^{*}\right)$ (with $A$ acting on $L^{2}\left(\mathbb{R}^{\mathbb{N}} ; \mathcal{H}\right)$ ) may be assumed cyclic for $U$, hence cyclic for $\mathbb{V}$.

We must finally show that $R\left(C^{1 / 2}\right)=R\left(A^{*}\right)$. We have

$$
R\left(A^{*}\right)=\bigcup_{k} R\left(A_{k}^{*}\right)=\bigcup_{k} R\left(C_{k}^{1 / 2}\right)
$$

and

$$
R(C)=\bigcup_{k} R\left(C_{k}\right)
$$

As $R\left(A^{*}\right)=R\left(A^{*} A\right), R\left(C^{1 / 2}\right)=R(C)$ and we have shown that $R\left(C^{1 / 2}\right)=$ $R\left(A^{*}\right)$ as required.

Finally, we wish to make the connection with our original aim-to show that Hamiltonians of the form

$$
H(t)=H_{0}+\sum_{k=1}^{N} \lambda_{k}\left|\psi_{k}\right\rangle\left\langle\psi_{k}\right| \sum_{n=0}^{\infty} \delta(t-n T)
$$

have a pure point quasi-energy spectrum.

THEOREM IV.4 Let $H_{0}$ be pure point, and define our time-dependent Hamiltonian as in (47). If $\psi_{1}, \ldots, \psi_{N} \in l_{1}\left(H_{0}\right)$, then for a.e. $\lambda=\left(\lambda_{1}, \ldots, \lambda_{N}\right)$ in $\mathbb{R}^{N}$, the Floquet operator

$$
V=e^{i\left(\sum_{k=1}^{N} \lambda_{k}\left|\psi_{k}\right\rangle\left\langle\psi_{k}\right|\right) / \hbar} U
$$

has pure point spectrum.

Proof. This theorem is just a special case of Theorem IV.3 with the $A_{k}$ s given by $\left|\psi_{k}\right\rangle\left\langle\psi_{k}\right|$. Noting (7), the $A_{k}$ s clearly commute. As Howland shows, $|\psi\rangle\langle\psi|$ is strongly $H$-finite if and only if $\psi \in l_{1}\left(H_{0}\right)$. Thus Theorem IV.3 applies and the result follows. 


\section{Discussion of results and potential applications}

Of fundamental importance in showing that the quasi-energy spectrum remains pure point for a.e. perturbation strength $\lambda$, was the fact that $\psi_{k} \in l_{1}\left(H_{0}\right)$. That is, if we write

$$
\left|\psi_{k}\right\rangle=\sum_{n=0}^{\infty}\left(a_{k}\right)_{n}\left|\phi_{n}\right\rangle
$$

where the $\left|\phi_{n}\right\rangle$ are the basis states of $H_{0}$, then $\psi_{k} \in l_{1}\left(H_{0}\right)$ if and only if

$$
\sum_{n=0}^{\infty}\left|\left(a_{k}\right)_{n}\right|<\infty .
$$

If this requirement is dropped, and we only retain $\psi_{k} \in l_{2}\left(H_{0}\right)$, then Theorem 3.1 in [2] fails and there is the possibility that $V(\lambda)$ will have a non empty continuous spectrum. It was this fact that Milek and Seba [10] took advantage of in showing that the rank-1 kicked rotor could contain a singularly continuous spectral component under certain conditions on the ratio of the kicking frequency and the fundamental rotor frequency. They analysed two regimes of the perturbation. One where $\psi \in l_{1}\left(H_{0}\right)$, in which case the numerical results clearly showed pure point recurrent behaviour, and the other where $\psi \in l_{2}\left(H_{0}\right)$, but $\psi \notin l_{1}\left(H_{0}\right)$. In the second case, the authors further proved that the absolutely continuous part of the spectrum was empty, and thus the system contained a singularly continuous spectral component. The numerical results reflected this, with a diffusive type energy growth being observed.

With the generalisation of Combescure's work here, namely our Theorem IV.4 it should now be possible to investigate the full class of rank-N kicked Hamiltonians. A sufficient requirement for recurrent behaviour has been shown to be $\psi_{k} \in l_{1}\left(H_{0}\right)$ and so we must turn our attention to perturbations where this requirement is no longer satisfied.

The challenge will be of course to find systems for which one can show that the absolutely continuous part of the spectrum is empty. Such systems would be candidates for classification as quantum chaotic systems.

\section{Acknowledgements}

This work was supported by the Australian Research Council. 


\section{References}

[1] M. Combescure. Spectral Properties of a Periodically Kicked Quantum Hamiltonian. J. Statist. Phys., 59:679, 1990.

[2] James S. Howland. Perturbation Theory of Dense Point Spectra. J. Func. Anal., 74:52, 1987.

[3] James S. Howland. Stationary Scattering Theory for Time-dependent Hamiltonians. Math. Ann., 207:315, 1974.

[4] James S. Howland. Scattering Theory for Hamiltonians Periodic in Time. Indiana Univ. Math. J., 28(3):471, 1979.

[5] James S. Howland. Floquet Operators with Singular Spectrum. I. Ann. Inst. H. Poinc. Phys., 49:309, 1989.

[6] L. Bunimovich, H. R. Jauslin, J. L. Lebowitz, A. Pellegrinotti, and P. Nielaba. Diffusive Energy Growth in Classical and Quantum Driven Oscillators. J. Statist. Phys., 62(3/4):793, 1991.

[7] Barry Simon and Tom Wolff. Singular Continuous Spectrum under Rank One Perturbations and Localization for Random Hamiltonians. Commun. Pure Appl. Math., 39:75, 1986.

[8] M. Hossein Partovi. Absence of sensitivity to initial conditions in quantum dynamics. Phys. Rev. A, 45(2):R555, January 1992.

[9] Michael Reed and Barry Simon. Methods of modern mathematical physics, volume 1: Functional analysis. Academic Press, New York, 1972.

[10] B. Milek and P. Seba. Singular continuous quasienergy spectrum in the kicked rotator with separable perturbation: Possibility of the onset of quantum chaos. Phys. Rev. A, 42(6):3213, September 1990.

[11] Y. Last. Quantum Dynamics and Decompositions of Singular Continuous Spectra. J. Func. Anal., 142(2):406, December 1995.

[12] Michael Reed and Barry Simon. Methods of modern mathematical physics, volume 3: Scattering theory. Academic Press, New York, 1979.

[13] J. M. Combes. Connections Between Quantum Dynamics and Spectral Properties of Time-Evolution Operators. In Ames et al. [30], page 59. 
[14] Kenji Yajima and Hitoshi Kitada. Bound states and scattering states for time periodic Hamiltonians. Ann. Inst. H. Poinc. Phys., 39(2):145, 1983.

[15] I. Antoniou and Z. Suchanecki. Quantum systems with fractal spectra. Chaos Solitons Fractals, 14:799, 2002.

[16] I. Antoniou and Z. Suchanecki. On Computability of Decaying and Nondecaying States in Quantum Systems with Cantor Spectra. Int. J. Theor. Phys., 42(10):2255, 2003.

[17] Michael Reed and Barry Simon. Methods of modern mathematical physics, volume 4: Analysis of operators. Academic Press, New York, 1978.

[18] Tosio Kato. Perturbation Theory for Linear Operators. Springer-Verlag, Berlin, 2nd edition, 1976.

[19] I.S. Gradshteyn and I.M. Ryzhik. Table Of Integrals, Series, And Products. Academic Press, New York, 1980.

[20] S. Saks. Theory of the Integral. Z Subwencji Funduszu Kultury Narodowej, Warsaw, 1937.

[21] Sir Harold Jeffreys and Bertha (Lady Jeffreys) Swirles. Methods of mathematical physics. Cambridge University Press, Cambridge, 3rd edition, 1962.

[22] Walter Rudin. Functional Analysis. Tata McGraw-Hill Publishing Company, New Delhi, 1974.

[23] George Bachman and Lawrence Narici. Functional Analysis. Academic Press, New York, 1972.

[24] Louis de Branges. Perturbations of Self-Adjoint Transformations. Am. J. Math., 84:543, 1962.

[25] Tosio Kato and S. T. Kuroda. The Abstract Theory of Scattering. Rocky Mt. J. Math., 1(1):127, 1971.

[26] P. Dienes. The Taylor Series. Dover Publications, Inc., New York, 1957.

[27] A. Erdelyi. Tables of Laplace Transforms, volume 1. McGraw Hill, New York, 1954.

[28] Frigyes Riesz and Béla Szokefalvi-Nagy. Functional Analysis. Frederick Ungar Pub. Co., New York, 1955. 
[29] Walter Rudin. Real \& Complex Analysis. Tata McGraw-Hill Publishing Company, New Delhi, 1974.

[30] W. F. Ames, E. M. Harrell II, and J. V. Herod, editors. Differential Equations with Applications to Mathematical Physics, volume 192 of Mathematics in Science and Engineering, Boston, 1993. Academic Press. 